\title{
Massive neutral gauge boson production as a probe of nuclear modifications of parton distributions at the LHC
}

\author{
${ }^{a}$ Vadim Guzey, ${ }^{b, c}$ Marco Guzzi, ${ }^{b}$ Pavel M. Nadolsky, \\ ${ }^{d}$ Mark Strikman, and ${ }^{b}$ Bowen Wang \\ a Department of Physics, Hampton University, \\ Hampton, VA 23668, USA \\ ${ }^{b}$ Department of Physics, Southern Methodist University, \\ Dallas, TX 75275, USA \\ ${ }^{c}$ Deutsches Elektronensynchrotron DESY, \\ Notkestrasse 85 D-22607 Hamburg, Germany \\ ${ }^{d}$ Department of Physics, The Pennsylvania State University, \\ State College, PA 16802, USA
}

\begin{abstract}
We analyze the role of nuclear modifications of parton distributions, notably, the nuclear shadowing and antishadowing corrections, in production of lepton pairs from decays of neutral $Z$ and $\gamma^{*}$ gauge bosons in proton-lead and lead-collisions at the LHC. Using the Collins-Soper-Sterman resummation formalism that we extended to the case of nuclear parton distributions, we observed a direct correlation between the predicted behavior of the transverse momentum and rapidity distributions of the produced vector bosons and the pattern of quark and gluon nuclear modifications. This makes production of $Z / \gamma^{*}$ in $p A$ and $A A$ collisions at the LHC a useful tool for constraining nuclear PDFs in the small- $x$ shadowing and moderate- $x$ antishadowing regions.
\end{abstract}




\section{Introduction}

Nuclear modifications of structure functions and parton distribution functions (PDFs) are a firmly established phenomenon extensively studied during last three decades [1, 2, 3, 4, 5, 6]. Presenting the emerging pattern of these modifications in terms of the ratio of the nuclear to free nucleon structure functions, $R\left(F_{2}\right) \equiv F_{2 A}(x) /\left[A F_{2 N}(x)\right]$, one observes the following characteristic trend in different regions of Bjorken $x: R\left(F_{2}\right)<1$ for $x \leq 0.05-0.1$ (nuclear shadowing), $R\left(F_{2}\right)>1$ for $0.1 \leq x \leq 0.3$ (antishadowing), $R\left(F_{2}\right)<1$ for $0.3 \leq x \leq 0.8$ (the EMC effect), and $R\left(F_{2}\right)>1$ for $x>0.8$ (Fermi motion). The four nuclear effects that are responsible for the medium modifications of the PDFs have different magnitudes and patterns. Indeed, the suppression due to nuclear shadowing increases with the atomic number $A$ and could be as large as, e.g., $20 \%$ for ${ }^{40} \mathrm{Ca}$; the enhancement due to antishadowing is a few-percent effect that does not reveal a distinctive $A$ dependence; the EMC effect suppression increases with $A$ and reaches, e.g., $20 \%$ around $x=0.65$ for ${ }^{56} \mathrm{Fe}$; the enhancement of $R\left(F_{2}\right)$ due to the nucleon Fermi motion inside nuclei for $x>0.8$ increases with $x$, becomes as large as $20-30 \%$ for $x \sim 0.9$, and leads to a nonvanishing nuclear PDF at $x>1$.

In the leading-twist approach, this pattern of nuclear modifications of $R\left(F_{2}\right)$ translates into a similar picture of medium modifications of PDFs [7, 8, 9, 10, 11]. However, the magnitude of nuclear modifications of individual quark and gluon distributions in nuclei in different regions of the light-cone fraction $x$ is rather uncertain. This is especially true for the gluon channel, as the indirect extraction of the gluon distribution from mostly fixed-target experiments on deep inelastic scattering (DIS) with nuclear targets leaves the gluon distribution in nuclei largely unconstrained.

In this paper we examine the possibility of establishing additional constraints on nuclear PDFs, primarily in the small- $x$ nuclear shadowing region, by analyzing the size of nuclear corrections in neutral gauge boson production in proton-lead and lead-lead reactions at the Large Hadron Collider (LHC). In particular, we study the transverse momentum distribution of $Z / \gamma^{*}$ production with its subsequent decay into a lepton pair in the reaction $A+B \rightarrow\left(Z / \gamma^{*} \rightarrow \ell \bar{\ell}\right)+X$, with $A=p$ or ${ }^{208} \mathrm{~Pb}, B={ }^{208} \mathrm{~Pb}$, and $\ell=e$ or $\mu$, by using the Collins-Soper-Sterman (CSS) formalism [12, 13, 14, 15] implemented in the resummation computer code RESBos [16, 17]. For this computation, we used the nuclear PDFs described in Sec. 3 and carried out the resummation calculation with the resulting nuclear parton distributions. The choice of this channel presents several advantages: it is very clean, has a high rate and is very well understood in proton-proton collisions. Moreover, the $Q_{T}$ distribution of heavy vector bosons can be easily reconstructed experimentally and, thus, can be measured very precisely. Access to a wide range of boson 
virtualities provides a powerful test of the leading-twist factorization and QCD evolution.

The extension of the CSS resummation formalism to nuclear scattering is described in Sec. 2, The treatment of nuclear corrections in the leading-twist approach is reviewed in Sec. 3, while Sec. 4 presents numerical predictions for nuclear corrections in $Z / \gamma^{*}$ production in $p A$ and $A A$ scattering at the LHC, in two distinct intervals of the Drell-Yan pair mass, $70<Q<110$ $\mathrm{GeV}$ and $5<Q<20 \mathrm{GeV}$. We examine nuclear corrections to the Drell-Yan rapidity and transverse momentum distributions and discuss dependence on the nonperturbative $k_{T}$ smearing corrections. Finally, Sec. 5 contains our conclusions.

\section{Application of $Q_{T}$ resummation to nuclear scattering}

\section{$2.1 \quad p p$ collisions}

In the standard framework [12, 13, 14, 15] for transverse momentum resummation in nucleonnucleon collisions, the differential cross section for vector boson production reads

$$
\begin{aligned}
\frac{d \sigma}{d Q^{2} d y d q_{T}^{2}} & =\int \frac{d^{2} \vec{b}}{(2 \pi)^{2}} e^{i \vec{q}_{T} \cdot \vec{b}} \widetilde{W}(b, Q, y)= \\
& =\int_{0}^{\infty} \frac{b d b}{2 \pi} J_{0}\left(q_{T} b\right) \widetilde{W}^{\text {pert }}\left(b_{*}, Q, y\right) \widetilde{W}^{N P}(b, Q, y)+Y\left(Q, Q_{T}, y\right),
\end{aligned}
$$

where $Q, Q_{T}$, and $y$ are the invariant mass, transverse momentum, and rapidity of the vector boson, respectively. The cross section consists of two terms, a Fourier-Bessel integral over the transverse position $(b)$ that evaluates the resummed logarithmic contributions in the limit $Q_{T}^{2} \ll Q^{2}$, and the finite piece $Y\left(Q, Q_{T}, y\right)$ that dominates at $Q_{T}^{2} \approx Q^{2}$. The resummed form factor $\widetilde{W}(b, Q, y)$ receives leading-power and power-suppressed contributions, and those are incorporated into the resummation integral of Eq. (1) as $\widetilde{W}^{\text {pert }}$ and $\widetilde{W}^{N P}$, respectively.

The form factor $\widetilde{W}^{\text {pert }}$ and non-singular contribution $Y$ (defined as the difference between the fixed-order calculation and the approximation for the perturbative part $\widetilde{W}^{\text {pert }}$ to the same order in $\alpha_{s}$ ) are computed in perturbative QCD. The explicit expression for $\widetilde{W}^{\text {pert }}$ is

$$
\begin{aligned}
\widetilde{W}^{p e r t} & =\sum_{j=u, d, s \ldots} \sigma_{j}^{(0)} e^{-\mathcal{S}\left(b, Q, C_{1}, C_{2}\right)} \\
& \times \sum_{a=g, q}\left[\mathcal{C}_{j a} \otimes f_{a / h_{1}}\right]\left(x_{1}, \frac{C_{1}}{C_{2}}, \frac{C_{3}}{b}\right) \sum_{b=g, q}\left[\mathcal{C}_{\bar{j} b} \otimes f_{b / h_{2}}\right]\left(x_{2}, \frac{C_{1}}{C_{2}}, \frac{C_{3}}{b}\right)
\end{aligned}
$$

in terms of the Sudakov exponential

$$
e^{-\mathcal{S}\left(b, Q, C_{1}, C_{2}\right)}=\exp \left[-\int_{C_{1}^{2} / b^{2}}^{C_{2}^{2} Q^{2}} \frac{d \bar{\mu}^{2}}{\bar{\mu}^{2}} \mathcal{A}\left(\bar{\mu} ; C_{1}\right) \ln \left(\frac{C_{2}^{2} Q^{2}}{\bar{\mu}^{2}}\right)+\mathcal{B}\left(\bar{\mu} ; C_{1}, C_{2}\right)\right]
$$


and convolutions $\left[\mathcal{C}_{j / a} \otimes f_{a / h}\right]$ of Wilson coefficient functions $\mathcal{C}_{j / a}\left(z, C_{1} / C_{2}, \mu_{F}=C_{3} / b\right)$ with distributions $f_{a / h}\left(z, \mu_{F}\right)$ of partons $a$ inside the initial-state hadron $h$. The normalization factor is

$$
\begin{aligned}
\sigma_{j}^{(0)} & \equiv \frac{M_{Z}^{2} G_{F}^{2}}{12 N_{c} S} \frac{Q^{2}}{\left(Q^{2}-M_{Z}^{2}\right)^{2}+M_{Z}^{2} \Gamma_{Z}^{2}}\left[\left(1-4\left|e_{j}\right| \sin ^{2} \theta_{w}\right)^{2}+1\right] \\
& \times\left[\left(1 / 2-\sin ^{2} \theta_{w}\right)^{2}+\sin ^{4} \theta_{w}\right]
\end{aligned}
$$

in $p p \rightarrow\left(Z^{0} \rightarrow e^{+} e^{-}\right) X$, and

$$
\sigma_{j}^{(0)} \equiv \frac{1}{S} \frac{4 \pi^{2} \alpha_{E M}^{2}}{3 N_{c} Q^{2}} e_{j}^{2}
$$

in $p p \rightarrow\left(\gamma^{*} \rightarrow e^{+} e^{-}\right) X$. Here $M_{Z}$ is the $Z$ boson mass, $G_{F}$ is the Fermi constant, $\theta_{w}$ is the weak angle, $\sqrt{S}$ is the collision energy, $N_{c}=3$ is the number of colors, $\alpha_{E M}$ is the fine structure constant, and $e_{j}=2 / 3$ or $-1 / 3$ is the fractional quark charge. The scale dependence of $\widetilde{W}^{\text {pert }}$ is ascribed to three scale parameters $C_{1}=b \bar{\mu}, C_{2}=\bar{\mu} / Q$, where $\bar{\mu}$ is the integration variable in the Sudakov exponent, and $C_{3}=\mu_{F} b$, where $\mu_{F}$ is the factorization scale in the convolutions $\left[\mathcal{C}_{j / a} \otimes f_{a / h}\right]$ with the PDFs.

The function $\widetilde{W}^{N P}(b, Q)$ in Eq. (1) provides a model for power-suppressed terms in the resummed form factor that play an important role at transverse positions of order $1 \mathrm{GeV}^{-1}$ or above. We evaluate the perturbative part $\widetilde{W}^{\text {pert }}$ as a function of $b_{*}=b / \sqrt{1+b^{2} / b_{\max }^{2}}$ and combine with the nonperturbative function $\widetilde{W}^{N P}(b, Q)$. The $b_{*}$ variable [13, 14] realizes a smooth transition from the leading-power terms that dominate at small $b$ to nonperturbative dynamics that is important at large $b$. We implement the $b_{*}$ convention in its extended version [18] that can simulate various trends in the behavior of $\widetilde{W}(b, Q)$ at $b>1 \mathrm{GeV}^{-1}$ suggested by the nonperturbative models [19, 20, 21, 22, 23, 24, while at the same time retaining the exact perturbative prediction for $\widetilde{W}(b, Q)$ at $b<1 \mathrm{GeV}^{-1}$.

Parameters of $\widetilde{W}^{N P}(b, Q)$ are constrained by a fit to the experimental data. To reproduce the observed $Q_{T}$ distributions in Drell-Yan process, it suffices to approximate $\widetilde{W}^{N P}(b, Q)$ by a Gaussian smearing factor $\exp \left[-a\left(Q, x_{1}, x_{2}\right) b^{2}\right]$, where the $b^{2}$ dependence is associated with the lowest power-suppressed contribution [25], and $a\left(Q, x_{1}, x_{2}\right)$ is found from the fit [26, 17]. A recent parametrization for $a(Q)$ from [18] describes well both the low- $Q$ pair and $Z$ production. Recently, constraints on $a(Q)$ at $Q \approx M_{Z}$ were updated [27, 28] using data on angular distributions $d \sigma / d \phi_{\eta}^{*}$ in $Z$ boson production at the Tevatron Run-2 [29]. This updated nonperturbative parametrization will be used in our simulations, as it provides excellent description of $Z$ boson $Q_{T}$ distributions in $p p$ collisions at the LHC. 


\section{$2.2 p A$ and $A A$ collisions}

The CSS resummation formalism can be adapted for describing nuclear collisions using the leading-twist approach discussed in the next section. Since the hard scattering contributions are identical in proton and nuclear collisions in the leading-twist approximation, it suffices to replace the proton PDFs $f_{a / h}\left(x, \mu_{F}\right)$ in all parts of the resummed cross section (1) by nuclear

PDFs $f_{a / A}\left(x, \mu_{F}\right)$ for each initial-state nucleus. The smearing function $\widetilde{W}^{N P}(b, Q)$ may also depend on the type of the nuclear projectile, for example, due to nuclear broadening effects [30], and can be modified by adjusting the $a$ parameter.

Following this approach, the resummed predictions can be obtained for any set of nuclear PDFs that is available [7, 8, 9, 10, 11]. In the next Section, we describe the FGS nuclear PDFs that will be used in our numerical simulations.

\section{Leading-twist nuclear shadowing in nuclear parton distribu- tions}

\subsection{Theory roundup}

Nuclear shadowing in hadron-nucleus and real photon-nucleus scattering is a firmly established experimental phenomenon. At high energies, the scattering cross section on a nuclear target is smaller than the sum of the cross sections on the individual nucleons comprising the nuclear target. In the nucleus rest frame, nuclear shadowing is explained as being an example of quantum mechanical destructive interference between the scattering amplitudes corresponding to the interaction with one, two, three, etc., nucleons [31]. The modern theory of nuclear shadowing is based on the connection between nuclear shadowing and diffraction [32, which can be understood [33] as a manifestation of unitarity reflected in the Abramovsky-Gribov-Kancheli (AGK) cutting rules [34]. The accuracy of the resulting theory of nuclear shadowing is rather high, with corrections at the level of no more than a few percent.

Turning to hard processes with nuclei, it was observed that the connection between shadowing and diffraction is also valid for any processes initiated by a hard probe and, in particular, for deep inelastic scattering (DIS) with nuclei. Combining this connection with QCD factorization theorems for inclusive and diffractive DIS, nuclear parton distribution functions (PDFs) for small $x(x<0.05)$ can be calculated. The approach based on this connection is called the leading-twist theory of nuclear shadowing [5, 33, 35, 36] and its predictions for nuclear PDFs will be used in the numerical analysis in this paper. 


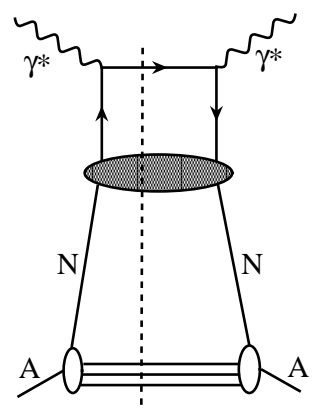

a)

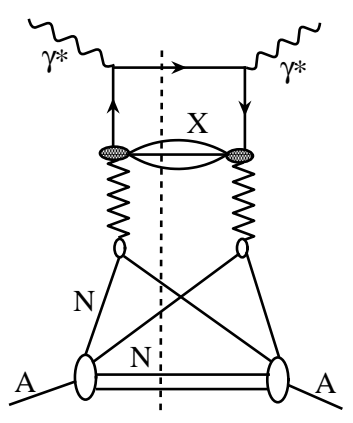

b)

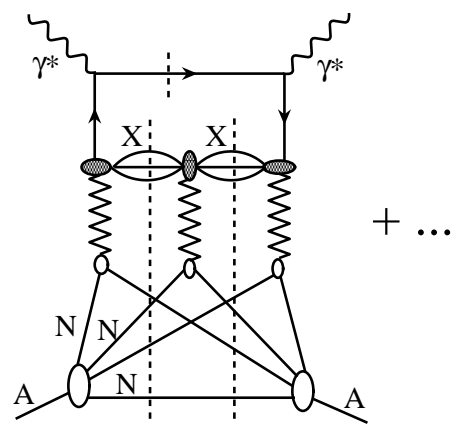

c)

Figure 1: Multiple scattering series for nuclear quark PDFs. Graphs $a, b$, and $c$ correspond to the interaction with one, two, and three nucleons, respectively. Graph $a$ gives the impulse approximation; graphs $b$ and $c$ contribute to the shadowing correction; the interaction with more than three nucleons is not shown but included in the final answer.

The modifications of nuclear parton distributions due to nuclear shadowing are given by a multiple scattering series, where each term corresponds to the interaction with one, two, three, etc. nucleons. In the graphical form, an example of such a series for the quark distributions in nuclei is presented in Fig. 1. The corresponding expression for the nuclear PDF of flavor $j$ at a certain initial scale $Q_{0}^{2}$ reads [36, 5]:

$$
\begin{aligned}
& x f_{j / A}\left(x, Q_{0}^{2}\right)=A x f_{j / N}\left(x, Q_{0}^{2}\right) \\
- & 8 \pi A(A-1) \Re e \frac{(1-i \eta)^{2}}{1+\eta^{2}} \int_{x}^{0.1} d x_{\mathbb{P}} \beta f_{j}^{D(4)}\left(\beta, Q_{0}^{2}, x_{\mathbb{P}}, t_{\min }\right) \int d^{2} b \int_{-\infty}^{\infty} d z_{1} \int_{z_{1}}^{\infty} d z_{2} \\
\times & \rho_{A}\left(\vec{b}, z_{1}\right) \rho_{A}\left(\vec{b}, z_{2}\right) e^{i\left(z_{1}-z_{2}\right) x_{\mathbb{P}} m_{n}} e^{-\frac{A}{2}(1-i \eta) \sigma_{\mathrm{soft}}^{j}\left(x, Q_{0}^{2}\right) \int_{z_{1}}^{z_{2}} d z^{\prime} \rho_{A}\left(\vec{b}, z^{\prime}\right)},
\end{aligned}
$$

where $f_{j}^{D(4)}$ is the diffractive parton distribution of the nucleon; $\rho_{A}$ is the nuclear matter density; $\eta$ is the ratio of the real to imaginary parts of the elementary diffractive amplitude, $\eta=\Re e A^{\text {diff }} / \Im m A^{\text {diff }} \approx 0.17$. The diffractive $\operatorname{PDF} f_{j}^{D(4)}$ depends on two light-cone fractions $x_{\mathbb{P}}=\left(M_{X}^{2}+Q^{2}\right) /\left(W^{2}+Q^{2}\right)$ and $\beta=x / x_{\mathbb{P}}$, and the invariant momentum transfer $t$, where $W$ is the invariant virtual photon-nucleon energy, $W^{2}=(q+p)^{2}$, and $M_{X}^{2}$ is the invariant mass squared of the produced intermediate diffractive state, denoted as "X" in Fig. 1. The longitudinal (collinear with the direction of the photon momentum) coordinates $z_{1}$ and $z_{2}$ and the transverse coordinate (impact parameter) $\vec{b}$ refer to the two interacting nucleons; $m_{n}$ is the nucleon mass. The $t$ dependence of $f_{j}^{D(4)}$ can be safely neglected as compared to the strong fall-off of the nuclear form-factor for $A>4$. As a result, $f_{j}^{D(4)}$ enters Eq. (6) at $t_{\min } \approx-x^{2} m_{n}^{2}\left(1+M_{X}^{2} / Q^{2}\right)^{2}$, and all nucleons enter with the same impact parameter $\vec{b}$. 
In Fig. 1]and in Eq. (6), modification of nuclear PDFs due to the interaction with two nucleons is calculated in a model-independent way through the nucleon diffractive PDFs, similarly to the case of hadron-deuteron scattering in the Gribov-Glauber theory of shadowing in soft processes. The contribution to nuclear shadowing from the interactions with $n \geq 3$ nucleons requires additional model-dependent considerations, since the interaction of a hard probe (virtual photon) with $n \geq 3$ nucleons is sensitive to fine details of the diffractive dynamics. Noticing that the analysis of diffraction in DIS implies that hadronic fluctuations of the virtual photon have predominantly large sizes, one can reliably parameterize the strength of the interaction with $n \geq 3$ nucleons by a single effective hadron-like cross section $\sigma_{\text {soft }}^{j}$. It was argued in Ref. [5] that the magnitude of $\sigma_{\text {soft }}^{j}$ can be reliably estimated using either the color dipole model or the model for hadronic fluctuations of the pion. As a result, using these two approaches one effectively obtains the lower and upper boundaries on $\sigma_{\text {soft }}^{j}$, which in turn correspond to the upper (FGS10_H) and lower (FGS10_L) boundaries on the predicted nuclear shadowing.

Note that Eq. (6) defines $x f_{j / A}\left(x, Q_{0}^{2}\right)$ only for the shadowing region of $x \leq 0.1$ and at $Q_{0}=$ $2 \mathrm{GeV}$ [5]. In the $0.03 \leq x \leq 0.2$ interval, there was introduced an additional effect of nuclear antishadowing (enhancement) in the gluon channel and modeled by requiring conservation of the momentum sum rule. Finally, while it was assumed that in the gluon and sea quark channels $x f_{j / A}\left(x, Q_{0}^{2}\right)=A x f_{j / N}\left(x, Q_{0}^{2}\right)$ for $x>0.2$, the valence quarks are subject to all four types of nuclear modifications briefly discussed in the introduction. As a consequence of the DGLAP $Q$ evolution from $Q_{0}$ to $Q>Q_{0}$, the large- $x$ nuclear effects, notably, the EMC effect, start also affecting the gluon and sea quark nuclear PDFs, see Figs. 2 and 3.

\subsection{Practical implementation}

We write a nuclear PDF as

$$
f_{j / A}\left(x, Q^{2}\right) \equiv R_{j}\left(x, Q^{2}\right) \cdot\left(A f_{j / N}\left(x, Q^{2}\right)\right),
$$

where $R_{j}\left(x, Q^{2}\right)$ is the ratio given by the leading-twist theory of nuclear corrections; $f_{j / N}$ is the free nucleon PDF. For $j=u, \bar{u}, d$, and $\bar{d}$ we have

$$
A f_{j / N}\left(x, Q^{2}\right) \equiv Z f_{j / p}\left(x, Q^{2}\right)+(A-Z) f_{j / n}\left(x, Q^{2}\right),
$$

where $A$ is the atomic number, $Z$ is the number of protons, $A-Z$ is the number of neutrons. The subscripts $p$ and $n$ indicate a proton and a neutron, respectively. The PDFs for the neutron for $u$ and $d$ (anti-)quarks are obtained via charge symmetry, $f_{u / n}\left(x, Q^{2}\right)=f_{d / p}\left(x, Q^{2}\right)$, etc. For heavier quarks and gluons, we have

$$
f_{j / N}\left(x, Q^{2}\right)=f_{j / p}\left(x, Q^{2}\right), \quad j=s, \bar{s}, c, \bar{c}, b, \bar{b}, g .
$$


Examples of resulting predictions of the leading-twist theory of nuclear corrections are presented in Figs. 2 and 3 , where the ratios of the next-to-leading order (NLO) nuclear $\left({ }^{208} \mathrm{~Pb}\right)$ to nucleon PDFs, $f_{j / A}\left(x, Q^{2}\right) /\left[A f_{j / N}\left(x, Q^{2}\right)\right]$, is plotted as functions of Bjorken $x$ at $Q=8$ and 90 $\mathrm{GeV}$, the representative virtualities of the $\gamma^{*} / Z$ boson encountered in our numerical calculation. The two sets of curves (labeled FGS10_H and FGS10_L) correspond to the two scenarios for $\sigma_{\text {soft }}^{j}$ (nuclear shadowing) that we have mentioned above. At $Q$ values shown in the figures, one predicts significant medium modifications of nuclear PDFs for each parton flavor: large shadowing (up to $30-40 \%$ ) for $x \lesssim 10^{-2}$, antishadowing at $0.05 \lesssim x \lesssim 0.15$, and the suppression originating from the EMC effect for $x \gtrsim 0.3$ values. The spread between the solid and dotted curves in Figs. 2 and 3 is the theoretical uncertainty of the present approach to nuclear PDFs; it is less than $10 \%$ for $A \sim 200$ and is much smaller for light nuclei.

\subsection{Experimental tests in the Drell-Yan process}

Next, we wish to outline how the $x$ dependence of the nuclear correction can be investigated experimentally by taking advantage of the basic kinematics of the Drell-Yan process. The hadronic cross section $\sigma$ for Drell-Yan pair production involves convolutions over longitudinal momentum fractions $\xi_{1}, \xi_{2}$ of the hard scattering cross section $\widehat{\sigma}$ with the PDFs,

$$
\begin{aligned}
\frac{d \sigma}{d Q^{2} d y d Q_{T}^{2}} & =\sum_{a, b} \int_{0}^{1} d \xi_{1} \int_{0}^{1} d \xi_{2} \frac{d \hat{\sigma}}{d Q^{2} d y d Q_{T}^{2}} f_{a / A}\left(\xi_{1}\right) f_{b / B}\left(\xi_{2}\right) \\
& \equiv \int_{\xi_{1, \text { min }}}^{1} d \xi_{1} \int_{\xi_{2, \text { min }}}^{1} d \xi_{2} h\left(\xi_{1}, \xi_{2}\right) \delta\left[\left(\frac{\xi_{1}}{x_{1}}-1\right)\left(\frac{\xi_{2}}{x_{2}}-1\right)-\frac{Q_{T}^{2}}{M_{T}^{2}}\right]
\end{aligned}
$$

In the second line, the Dirac delta function is separated from all other terms, indicated collectively by $h\left(\xi_{1}, \xi_{2}\right)$. It arises due to energy-momentum conservation and constrains the integration variables $\xi_{1}$ and $\xi_{2}$ to lie on a hyperbolic integration contour satisfying $K_{1} K_{2}=Q_{T}^{2} / M_{T}^{2}$, where $K_{1,2}=\xi_{1,2} / x_{1,2}-1, x_{1,2}=\left(M_{T} / \sqrt{S}\right) \exp ( \pm y)$, and $M_{T}=\sqrt{Q^{2}+Q_{T}^{2}}$.

Although $\xi_{1}$ and $\xi_{2}$ are integrated over an extended range, a large part of the cross section is contributed from around the point $K_{1}=K_{2}=Q_{T} / M_{T}$, where both $\xi_{1}$ and $\xi_{2}$ are small enough, and the corresponding PDFs are large. At this point,

$$
\begin{aligned}
& \xi_{1} \approx x_{1 c} \equiv \tau_{e f f} e^{y} \\
& \xi_{2} \approx x_{2 c} \equiv \tau_{e f f} e^{-y}
\end{aligned}
$$

where

$$
\tau_{e f f} \equiv \frac{M_{T}+Q_{T}}{\sqrt{S}}
$$




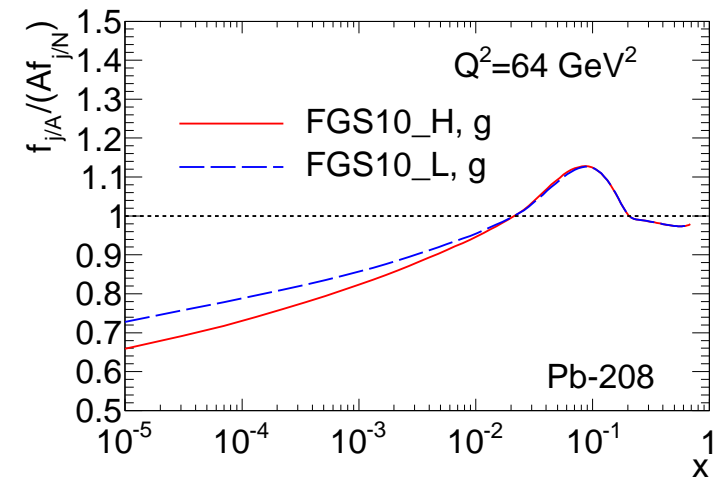

(a)

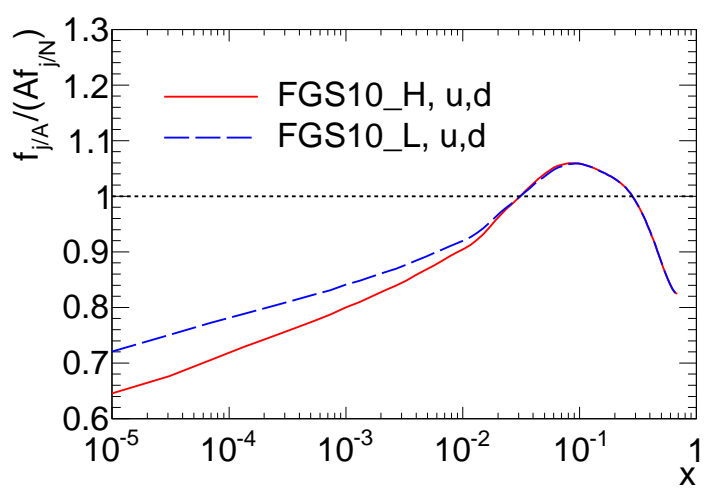

(c)

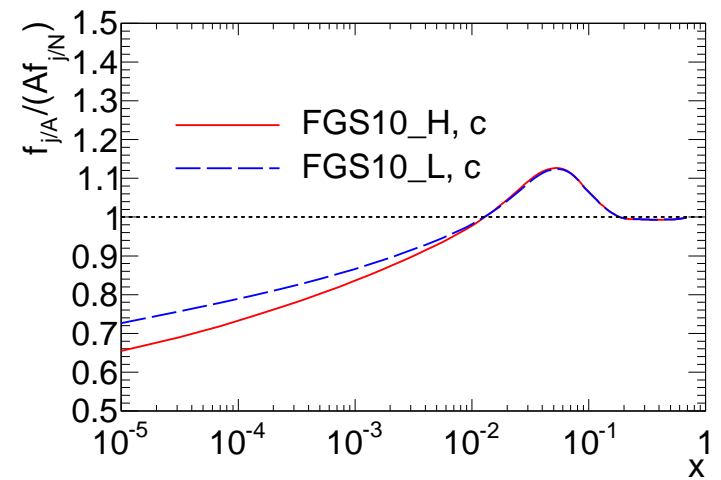

(b)

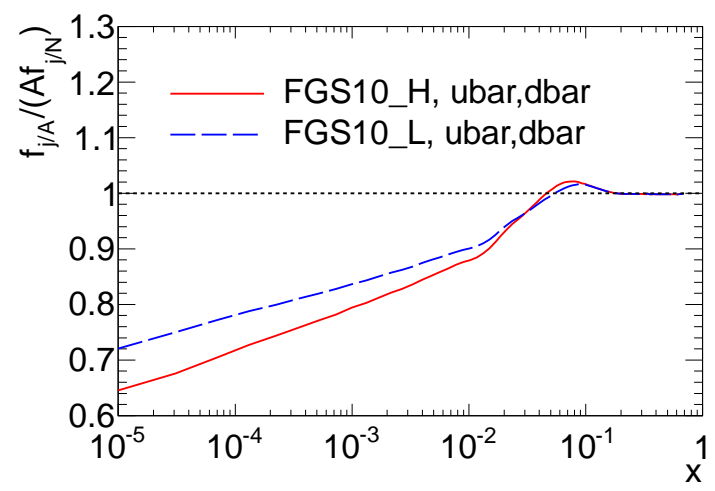

(d)

Figure 2: Predictions of the leading-twist theory of nuclear corrections for $f_{j / A}(x, Q) /\left[A f_{j / N}\left(x, Q^{2}\right)\right]$ for different partons in ${ }_{82}^{208} \mathrm{~Pb}$, with $Q=8 \mathrm{GeV}$, and for the indicated parton flavors. The two sets of curves correspond to the two scenarios of nuclear shadowing (see the text). 


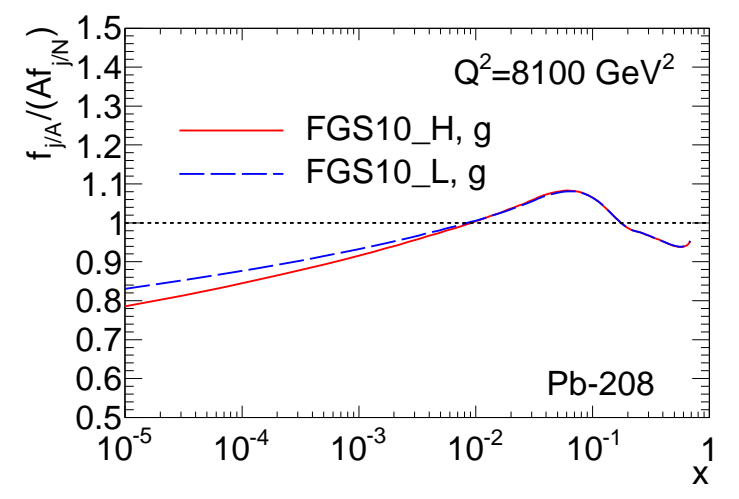

(a)

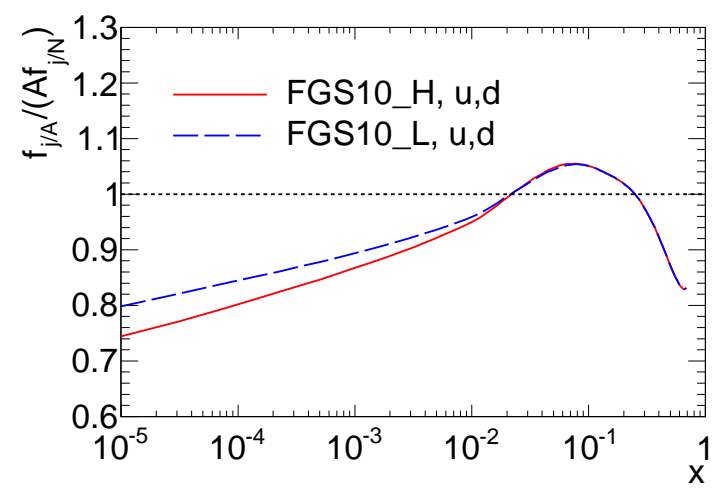

(c)

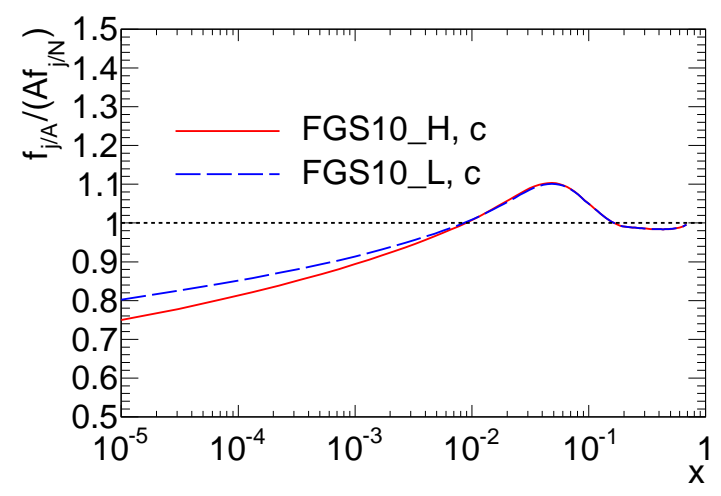

(b)

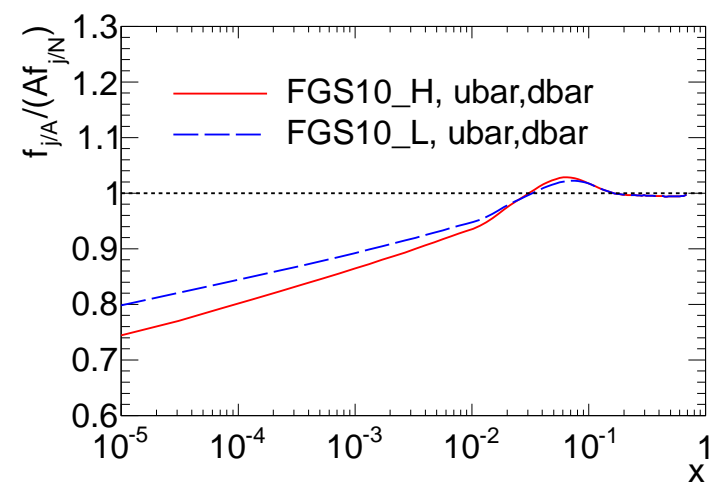

(d)

Figure 3: Same as Fig. 2, for $Q=90 \mathrm{GeV}$. 
Far away from this point, one of the PDFs is suppressed as $\xi \rightarrow 1$, and the cross section is reduced.

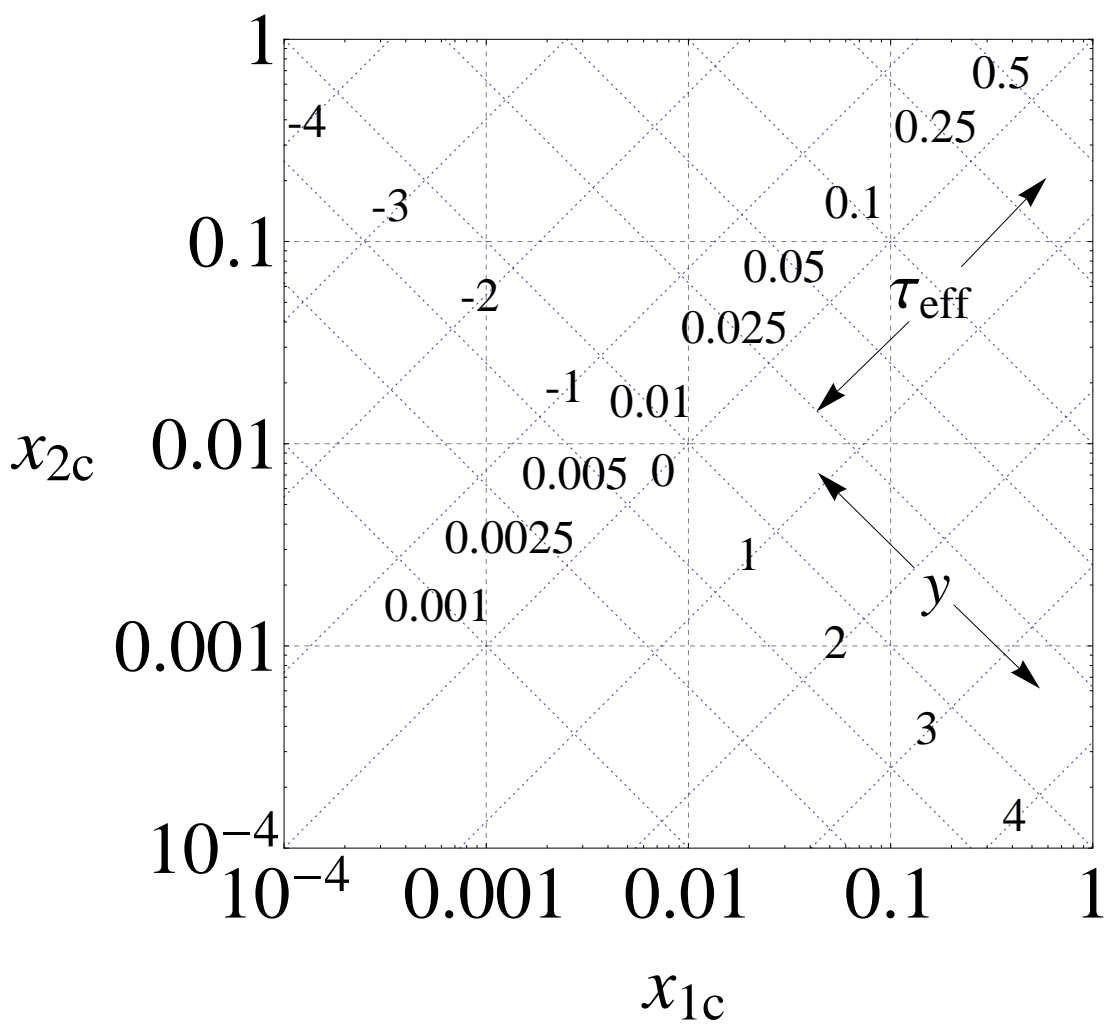

Figure 4: Representative momentum fractions $x_{1 c}$ and $x_{2 c}$ in initial-state particles 1 and 2 as functions of $y$ and $\tau_{\text {eff }}$.

The pair $\left\{x_{1 c}, x_{2 c}\right\}$ can thus characterize typical values of the momentum fractions in the initial-state hadrons 1 and 2 in most of contributing events for the given boson's 4-momentum. They can be evaluated from $\tau_{\text {eff }}$ and $y$ (or, equivalently, $\sqrt{S}, Q, Q_{T}$, and $y$ ) with the help of Eq. (11). A contour plot for conversion of $\left\{\tau_{e f f}, y\right\}$ into $\left\{x_{1 c}, x_{2 c}\right\}$ is presented in Fig. 4. Here, the contours indicate constant values of $\tau_{e f f}$ and $y$; the corresponding $x_{1 c}$ and $x_{2 c}$ values can be read off the horizontal and vertical axis.

For the following discussion, we also include Table 1 listing the ranges of $\tau_{\text {eff }}$ for the combinations of $\sqrt{S}, Q$ and $Q_{T}$ values typical for our figures.

We will argue in Sec. 4 that the behavior of the nuclear correction to the $Z / \gamma^{*}$ cross section can be understood qualitatively from the plots of the nuclear correction to the PDFs in Figs. 2 and 3, taking the $x_{1 c}$ and $x_{2 c}$ as the reference momentum fractions. The relation is accurate 


\begin{tabular}{|c|c|c|c|}
\hline$\sqrt{S}, \mathrm{TeV}$ & $Q, \mathrm{GeV}$ & $Q_{T}, \mathrm{GeV}$ & $\tau_{\text {eff }}$ \\
\hline \multirow{2}{*}{2.76} & 8 & $0-100$ & $0.0029-0.073$ \\
\cline { 2 - 4 } & 90 & $0-200$ & $0.033-0.15$ \\
\hline \multirow{2}{*}{4.4} & 8 & $0-100$ & $0.0018-0.046$ \\
\cline { 2 - 4 } & 90 & $0-200$ & $0.020-0.095$ \\
\hline \multirow{2}{*}{5.52} & 8 & $0-100$ & $0.0014-0.036$ \\
\cline { 2 - 4 } & 90 & $0-200$ & $0.016-0.08$ \\
\hline \multirow{2}{*}{8.8} & 8 & $0-100$ & $0.0009-0.023$ \\
\cline { 2 - 4 } & 90 & $0-200$ & $0.01-0.048$ \\
\hline
\end{tabular}

Table 1: Ranges of $\tau_{\text {eff }}$ values for kinematical regions shown in the figures.

within $5-10 \%$ and works better when small $\xi$ values dominate, since $h\left(\xi_{1}, \xi_{2}\right)$ in Eq. (10) is a monotonous function for small $\xi_{1,2}$. More detailed predictions require to know the behavior of $h\left(\xi_{1}, \xi_{2}\right)$ along the full integration range in Eq. (10). For the bulk of $Z$ bosons, $Q_{T}$ is much smaller than $Q$, and Eq. (11) reduces to the familiar

$$
\xi_{1,2} \approx x_{1,2}=(Q / \sqrt{S}) e^{ \pm y}
$$

However, Eq. (11) also clarifies the non-trivial $Q_{T}$ dependence of our numerical results, to which we turn next.

\section{Numerical predictions}

\subsection{Setup}

The FGS PDFs were implemented in the CSS resummation codes LEGACY and ResBos [16, 17] and used to compute Drell-Yan pair production cross sections in two characteristic mass ranges of $Q=70-110$ and $5-20 \mathrm{GeV}$ and for the boson rapidity $|y|<3.5$ corresponding to $x>10^{-5}$ at all considered energies. RESBOS performs resummation of the initial-state QCD radiation and decay of massive bosons along the lines described in Sec. 2. At $Q \approx M_{Z}$, we computed the dominant process of resonant $Z$ boson contribution, as well as small contributions from the continuous $\gamma^{*}$ background and $Z-\gamma^{*}$ interference. At $Q=5-20 \mathrm{GeV}$, we included the virtual photon contribution only, as the $Z$-mediated cross section is negligible in this mass range.

Nuclear PDFs are generated according to Eq. (7) using a CT10 NLO family of PDFs of the 
proton [37. To evaluate the nuclear correction to the differential cross section, we introduce the ratio

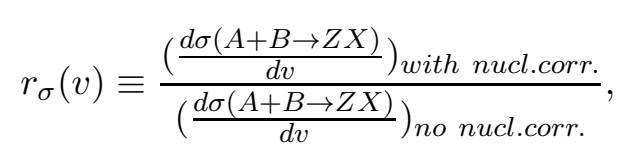

where $v=y$ or $Q_{T}$, in select ranges of $Q$ and/or $y$. The denominator is calculated assuming the absence of nuclear modifications of nuclear PDFs, i.e., according to Eq. (7) with $R_{j}\left(x, Q^{2}\right)=1$. The cross sections are computed for the following values of the center-of-mass energy at the LHC: $\sqrt{S_{p A}}=4.4 \mathrm{TeV}$ and $8.8 \mathrm{TeV}$ in the proton-lead scattering and $\sqrt{S_{A A}}=2.76 \mathrm{TeV}$ and $5.52 \mathrm{TeV}$ in the lead-lead scattering.

In the perturbative resummed form factor (2), the functions $\mathcal{A}, \mathcal{B}$, and $\mathcal{C}_{j / a}$ were evaluated to orders $\alpha_{s}^{3}, \alpha_{s}^{2}$, and $\alpha_{s}$, respectively [14, 38, 39, 40]. The scale parameters in $\widetilde{W}^{\text {pert }}$ were chosen as $\left\{C_{1}=C_{3}=2 b_{0}, C_{2}=2\right\}$. The nonperturbative contributions were introduced according to Ref. [28] using the $b_{*}$ convention with $b_{\max }=1.5 \mathrm{GeV}^{-1}$ and $\widetilde{W}^{N P}(b)=\exp \left[-a b^{2}\right]$. We take $a=1.1 \mathrm{GeV}^{2}$ by default in $Z$ production and $a=0.3$ or $1.1 \mathrm{GeV}^{2}$ in low- $Q$ Drell-Yan production. We assume the same $a$ value in the numerator and denominator of the ratio $r_{\sigma}$, so that the dependence on $a$ cancels well inside $r_{\sigma}$. The predictions for $r_{\sigma}$ that will be shown are hardly sensitive to the value of $a$ that is assumed.

The $Y$ term was estimated to NLO in photon-mediated subprocesses, and to NNLO in the pure $Z$ cross section by using the two-loop correction from Ref. [41]. The renormalization and factorization scales were set to $2 Q$ in the $Y$ piece. All these settings are in good agreement with ATLAS $Q_{T}$ distributions for $p p \rightarrow Z / \gamma^{*} X$ [42], as has been found in Ref. [28].

\subsection{Proton-lead collisions}

The $r_{\sigma}$ ratios for proton-lead collisions are shown in Figs. 5 5 for $Z$ boson production in a representative interval $70<Q<110 \mathrm{GeV}$ and in Figs. 8 [10 for low- $Q$ Drell-Yan process at $5<Q<20 \mathrm{GeV}$. The dashed purple line and short-dashed black line were computed using the FGS10_H and FGS10_L nuclear PDFs, respectively.

\subsection{1 $Z$ pole region}

We start with the plots of the $r_{\sigma}$ ratio for the $Z$ rapidity distribution in Fig. 5 , as they are the simplest. In $p A$ collisions, the nuclear correction acts on the PDF of the lead nucleus but not on the proton PDF. The nuclear correction depends on $\xi_{2}$, which is of order $x_{2 c}=\tau_{e f f} \exp (-y)$ for the most part. We can therefore understand the overall magnitude of the nuclear correction within $5-10 \%$ by estimating $x_{2 c}$ for the given $y$ and $Q_{T}$, as has been argued in Sec. 3.3 . 
Since $Q_{T}$ is much smaller than $Q$ for the majority of $Z$ events, a scan of $r_{\sigma}(y)$ over $y$ in Fig. 5 essentially translates into a scan over the momentum fraction $x$ in the PDF nuclear correction in Fig. 3. Negative (positive) values of $y$ correspond to large (small) values of $x=$ $\left(Q / \sqrt{S_{p A}}\right) \exp (-y)$. The typical $x$ value (equated to $x_{2 c}$ ) can be found for each $y$ from Fig. 4 and Table 1 .

In Fig. 3 we see the small- $x$ shadowing at $x \lesssim 2 \cdot 10^{-2}$, antishadowing at $2 \cdot 10^{-2} \lesssim x \lesssim 0.3$, and large- $x$ suppression at $x \gtrsim 0.3$. The exact boundaries between the three regions depend on the parton flavor. In the upper inset of Fig. 5, these regions translate into $r_{\sigma}<1$ at $y>0$, $r_{\sigma}>1$ at $-2.6<y<0$, and $r_{\sigma}<1$ for $y<-2.6$. The suppression is the most pronounced (of order 15-20\%) in the forward rapidity regions. The FGS10_L and FGS10_H predictions differ in the small- $x$ /large- $y$ shadowing region, but are very close otherwise.

In the lower inset (obtained for $\sqrt{S_{p A}}=8.8 \mathrm{TeV}$ ), we observe a qualitatively similar behavior of $r_{\sigma}$ as in the upper inset (for $\sqrt{S_{p A}}=4.4 \mathrm{TeV}$ ), but the antishadowing "hill" is shifted toward lower $y$ values, $-3.2 \lesssim y<-0.8$, as a result of reduced $\tau_{\text {eff }}$ for the same $Q$.

The measurement of $Q_{T}$ of the lepton pair provides an additional handle for probing the $x$ dependence. For $y$ fixed, a larger $Q_{T}$ value leads to a larger $\tau_{\text {eff }}$ and $x_{2 c}$ as compared to $Q_{T}=0$. Ranges of $\tau_{\text {eff }}$ in the $Q_{T}$ intervals shown in the figures are listed in Table 1 ,

We also observe that the flavor dependence of scattering contributions is distinct at small and large $Q_{T}$. At $Q_{T} \ll Q$, the scattering proceeds largely through $q \bar{q}$ annihilation, while at $Q_{T} \approx Q$ the $q g$ Compton scattering becomes competitive. The analysis of $Q_{T}$ dependence can thus identify nuclear corrections attributed to the gluon PDF.

The $Q_{T}$ dependent ratios $r_{\sigma}\left(Q_{T}\right)$ for $p A \rightarrow Z$ production are plotted at 4.4 and $8.8 \mathrm{TeV}$ in Figs. 6 and 7 in the specified bins of $y$. The $Q_{T}$ bins were chosen as in the $p p \rightarrow Z X$ measurements by ATLAS [42]. The rapidity dependence is not symmetric with respect to $y=0$ in the $p A$ case. Hence the $Q_{T}$ distributions are plotted separately for positive and negative $y$.

In both figures, the value of $r_{\sigma}$ at low $Q_{T}$ in each bin is in rough correspondence with the $r_{\sigma}(y)$ value for the corresponding $y$ in the rapidity distribution. This is expected, since most $Z$ events that contribute to the rapidity distribution have small $Q_{T}$. As $Q_{T}$ increases, the $r_{\sigma}\left(Q_{T}\right)$ changes non-trivially, especiall in the positive $y$ bins (right columns in the figures), where significant small- $x$ shadowing (of order 15-20\%) at $Q_{T} \rightarrow 0$ is superceded by antishadowing of up to $10 \%$ at $Q_{T}>50-100 \mathrm{GeV}$. 


\subsubsection{Low $Q$ region}

In the low invariant mass range, $5<Q<20 \mathrm{GeV}, Z$-mediated $e^{+} e^{-}$production is negligible compared to virtual photon contributions. In this case Table 1 and Fig. 4 tell us that the DrellYan process probes much smaller values of $x_{2, c}$, of order a few $10^{-3}$. The $r_{\sigma}$ rapidity distribution in Fig. 8 shows that small- $x$ nuclear shadowing dominates across the full rapidity range, with the exception of a small band of large negative rapidities below -2.5 . The magnitude of shadowing exhibits a monotonous increase from zero at $y \approx-2.5$ to $25-30 \%$ at $y \approx+3$.

Even when shadowing dominates across in some $y$ interval, antishadowing may still occur at this $y$ if $Q_{T}$ is high enough. This is illustrated by Figs. 9 and 10, showing $Q_{T}$ dependence of the $r_{\sigma}$ ratio in the low-mass range. In the bins with $y<0$ in the left columns of Figs. 9 and 10, $r_{\sigma}$ is smaller than 1 (larger than 1) at $Q_{T}=0\left(Q_{T}=20-30 \mathrm{GeV}\right)$. For $y<-3$, even large- $x$ suppression of up to $10 \%$ occurs at the largest $Q_{T}$ values close to $100 \mathrm{GeV}$.

At positive $y$ in Figs. 9 and 10 (right columns), small $Q_{T}$ shadowing is more prominent. In this $y$ region, corresponding to $x<2 \cdot 10^{-3}$ for $Q_{T}=0$, shadowing can reach $40 \%$ in the lowest $Q_{T}$ bins. As the transverse momentum increases above $Q_{T}=10 \mathrm{GeV}, r_{\sigma}$ remains below unity, in consistency with the $x_{2 c}$ value that is still quite small. Detailed variations of $r_{\sigma}$ are now more intricate, as a wide range of the momentum fractions $\xi_{2}$ contributes at these $y$ : $\xi_{2, \min } \leq \xi_{2} \leq 1$, where $\xi_{2, \min } \approx x_{2}$ is much smaller than 0.01 . As a result, $r_{\sigma}$ at $Q_{T}>10 \mathrm{GeV}$ shows both the dependence on the small- $x$ shadowing model (from $\xi_{2}<0.01$ ) and a "hill" from the antishadowing and enhancements in the valence PDFs at $\xi_{2}>0.01$.

It can be further shown that the $q g$ Compton scattering contributes of order $80 \%$ at the largest $Q_{T}$ shown in the figures. The Drell-Yan process at low $Q$ and large $Q_{T}$ is therefore an excellent probe of nuclear corrections to the nuclear PDFs, in full analogy with $p p$ case [43, 44].

\subsection{Numerical results for lead-lead collisions}

\subsection{1 $Z$ pole region}

The nuclear correction is more complex in lead-lead collisions, since both incoming beams contribute to the nuclear effects. In this case the rapidity distribution is symmetric, as can be seen in Fig. 11. The $\tau_{\text {eff }}$ values with $Q_{T}=0$ are equal approximately to 0.033 at $\sqrt{S_{A A}}=2.76$ $\mathrm{TeV}$ and 0.016 at $\sqrt{S_{A A}}=5.52 \mathrm{TeV}$ and correspond to mild antishadowing and small- $x$ shadowing, respectively. Consequently the $y=0$ region in Fig. 11] exhibits antishadowing of 2-3\% for $\sqrt{S_{A A}}=2.76 \mathrm{TeV}$ (upper inset) and $5 \%$ shadowing for $\sqrt{S_{A A}}=5.52 \mathrm{TeV}$ (lower inset). In the central region, predictions based on two nuclear models are almost indistinguishable. 
As $|y|$ increases, the strong small- $x$ shadowing in one of the initial-state nuclei overtakes the mild increase in $r_{\sigma}$ due to antishadowing associated with the second nuclei. At the largest rapidity shown $(|y| \rightarrow 3.5)$ the shadowing reaches up to $30 \%$ and has different magnitude in the two nuclear models.

$Q_{T}$ dependence of $r_{\sigma}$ is illustrated in Figs. 12 and 13, in three bins of $|y|$. We observe the strong small- $x$ shadowing (15-20\%) at the smallest $Q_{T}$ values, which is replaced by antishadowing (up to 10\%) at intermidiate $Q_{T}$, and eventually by large- $x$ suppression at the highest $Q_{T}$

attainable. The shadowing is generally stronger for $\sqrt{S_{A A}}=5.52 \mathrm{TeV}$ (Fig. 13) as a result of smaller typical $\tau_{e f f}$, of about 0.001 for $Q_{T}=0$ and 0.036 for $Q_{T}=100 \mathrm{GeV}$.

\subsubsection{Low- $Q$ region}

In the low invariant mass regime illustrated by Figs. 14,16, the shadowing is most pronounced; antishadowing in one nuclei never overcomes shadowing in the counterpart nuclei in either $y$ distribution or $Q_{T}$ distribution. Antishadowing produces characteristic bumplike features in the lower inset of the $y$ distribution in Fig. 14 and $Q_{T}$ distributions in Figs. 15 and 16, but never overcomes the overall suppression that results in $r_{\sigma}<1$. In the $Q_{T}$ distribution, the shadowing is generally stronger at the smallest $Q_{T}$ (up to $40 \%$ ) than in the $y$ distribution (25-30\%). The profile of $r_{\sigma}$ at the intermediate and large $Q_{T}$ value again displays a "hill", similarly to the $p A$ case.

\subsection{Nonperturbative smearing of the transverse momentum distribution}

One of the key uncertainties in determination of the nuclear PDF corrections from Drell-Yan $Q_{T}$ distributions is associated with the small- $Q_{T}$ nonperturbative function $\widetilde{W}^{N P}(b, Q)$ that is not completely known in nuclear scattering. In our analysis, $\widetilde{W}^{N P}(b, Q)$ has been determined from $p p$ collisions (cf. Sec. 2) and parametrized as

$$
\widetilde{W}^{N P}(b, Q)=\exp \left(-a(Q) b^{2}\right)
$$

with $a(Q)=1.1 \mathrm{GeV}^{2}$ at $Q \approx M_{Z}$ and $a=0.3-1.1 \mathrm{GeV}^{2}$ at low $Q$. In the previous subsections, the ratio $r_{\sigma}$ has quantified the effect of the nuclear correction to the nuclear PDF and was constructed to be almost independent of $a$. But for the actual $Q_{T}$ distributions, the nuclear modifications may also arise from the nonperturbative function $\widetilde{W}_{N P}(b, Q)$, which has impact on production of Drell-Yan pairs at $Q_{T}<20 \mathrm{GeV}$.

Analysis of the experimental data on Drell-Yan dilepton production in $p A$ collisions at $\sqrt{S}=$ $40 \mathrm{GeV}$ finds [45] a very small $A$-dependent broadening of the $Q_{T}$ distribution. At this energy 
(corresponding to $x \gtrsim 0.1$ ) and $Q_{T}<3 \mathrm{GeV}, \Delta\left\langle Q_{T}^{2}\right\rangle$ grows approximately linearly with $A^{1 / 3}$ and reaches $0.1 \mathrm{GeV}^{2}$ for the heaviest nuclei. Moreover the analysis indicates that the data at lower energies are consistent with the energy-independent $\Delta\left\langle Q_{T}^{2}\right\rangle$. Physically, the broadening of the $Q_{T}$ distribution can be interpreted as multiple rescattering of the incoming quark off $A^{1 / 3}$ nucleons. At smaller momentum fractions $\xi$, the broadening from rescattering may be larger due to the increase of the gluon density at small $x$. At the same time the $A$ dependence of the broadening effect may become weaker due to the leading-twist nuclear shadowing.

In the CSS resummation formalism, broadening is accommodated by modifying the nonperturbative $k_{T}$ distribution in the nucleus at small $x$ in the region of nuclear shadowing. The nuclear shadowing is stronger for small virtualities, so that effective $k_{T}^{2}$ at scale $1 \mathrm{GeV}$ may be somewhat larger than in the nucleon case.

To account for this possibility, and the fact that $a$ has uncertainties even in $p p$ collisions, we give the plots of $d \sigma / d Q_{T}$ for a rather wide range of $a$ in a representative case of lead-lead collisions at $\sqrt{S_{A A}}=2.76 \mathrm{TeV}$ in the shadowing model FGS10_H.

In Fig. 17 we compare predictions for $d \sigma / d Q_{T}$ at $5<Q<20 \mathrm{GeV}$ for the range $a=$ $0.3,0.7,1.1 ., 1.5$, and $1.9 \mathrm{GeV}^{2}$, divided by $d \sigma / d Q_{T}$ for $a=0.3 \mathrm{GeV}^{2}$. The value of $a=0.3 \mathrm{GeV}^{2}$ is of order of the average $a$ value in $p p$ collisions in this $Q$ interval in fixed-target (large- $x$ ) DrellYan production [18]. Larger $a$ values would reflect the increased nonperturbative $k_{T}$ in the nuclei and result in broader $Q_{T}$ distributions that may be more typical for small $x$ values [46] and/or nuclear targets. In this specific example, increasing $a$ from 0.3 to $1.9 \mathrm{GeV}^{2}$ increases the average $\left\langle Q_{T}^{2}\right\rangle$ in the affected interval $0<Q_{T}<20 \mathrm{GeV}$ by $2.5 \mathrm{GeV}^{2}$ (from 28.6 to $31.1 \mathrm{GeV}^{2}$, which is much larger than $\Delta\left\langle Q_{T}^{2}\right\rangle$ in a fixed-target experiment discussed above). The dependence of $\left\langle Q_{T}^{2}\right\rangle$ on a reflects the interplay of perturbative and nonperturbative QCD contributions and varies with $\sqrt{S}$ and $Q$.

Similarly, in Fig. 18 we show predictions for $d \sigma / d Q_{T}$ at $70<Q<110 \mathrm{GeV}$ for the range $a=0.7,1.1,1.5$, and $1.9 \mathrm{GeV}^{2}$, divided by $d \sigma / d Q_{T}$ for the nominal $a=1.1 \mathrm{GeV}^{2}$. The range $0.7-1.5 \mathrm{GeV}^{2}$ is of order of the current $95 \%$ experimental uncertainty in $a(Q)$ in $p p \rightarrow Z X$ [27]. The curve for $1.9 \mathrm{GeV}^{2}$ is an example of more extreme broadening than in $p p$ collisions. Note that $Q_{T}$ distributions in $Z$ production are generally less sensitive to the nonperturbative smearing than in low- $Q$ Drell-Yan process.

In the two figures, we observe that the strongest dependence on $a(Q)$ occurs at $Q_{T}<4 \mathrm{GeV}$, where the cross section varies between 80 and $150 \%$ (85 and 130\%) in the low- $Q$ (high- $Q$ ) region. The variations are much milder $( \pm 10 \%$ and $\pm 5 \%)$ at $4<Q_{T}<20 \mathrm{GeV}$ and practically vanish at $Q_{T}>20 \mathrm{GeV}$. We see that, while the nonperturbative smearing is relevant at $Q_{T}$ below 5 
$\mathrm{GeV}$, it leaves the $Q_{T}$ distributions intact at larger $Q_{T}$ and hence will not influence sensitivity to the nuclear PDF corrections in most of the $Q_{T}$ range.

\section{Conclusions}

We studied production of the neutral $Z$ and $\gamma^{*}$ gauge bosons with their subsequent decay into lepton pairs in proton-lead and lead-collisions at the LHC and examined the role of nuclear medium modifications of nuclear PDFs. By extending the REsBos code for the Collins-SoperSterman $Q_{T}$ resummation to the case of nuclear parton distributions, we analyzed the transverse momentum $Q_{T}$ and rapidity $y$ dependence of the production cross section. We examined $r_{\sigma}$, the ratio of the differential production cross sections with and without nuclear modifications of quark and gluon PDFs in nuclei, and found unambiguous correspondence between the predicted behavior of $r_{\sigma}$ and the pattern of nuclear modifications of nuclear PDFs. At central or moderate rapidities, there may be a region with $r_{\sigma}>1$ as a consequence of antishadowing of the valence quark distributions in nuclei. For forward and backward rapidities, we generally predict that $r_{\sigma}<1$ due to the suppression of the quark and gluon distributions in nuclei by nuclear shadowing. For large rapidities the ratio $r_{\sigma}$ can also discriminate between different scenarios (magnitudes) of nuclear corrections, while the large- $Q_{T}$ region probes the gluon nuclear correction. A variety of measurements that can be carried out with $p A$ and $A A$ Drell-Yan production make it an informative test of the QCD evolution in high-energy heavy-nuclei scattering and of the pattern of medium modifications of nuclear PDFs.

The ResBos code and resummed grids for simulations in this study can be downloaded from http://hep.pa.msu.edu/resum/."

\section{Acknowledgments}

M.G. and P.N. thank F. I. Olness and C.-P. Yuan for useful discussions. M. S. and P.N. thank organizers of the 2011 workshop "High-energy QCD after the start of the LHC" at the Galileo Galilei Institute in Florence, Italy for financial support and hospitality during the initial part of this work. This work was supported by the U.S. DOE Early Career Research Award de-sc0003870 and by the Lightner Sams Foundation.

\section{References}

[1] L. Frankfurt and M. Strikman, Phys.Rept. 160 (1988) 235. 
[2] M. Arneodo, Phys.Rept. 240 (1994) 301.

[3] D.F. Geesaman, K. Saito and A.W. Thomas, Ann.Rev.Nucl.Part.Sci. 45 (1995) 337.

[4] G. Piller and W. Weise, Phys.Rept. 330 (2000) 1, hep-ph/9908230.

[5] L. Frankfurt, V. Guzey and M. Strikman, Phys.Rept. 512 (2012) 255, arXiv:1106.2091.

[6] L. Frankfurt and M. Strikman, Int.J.Mod.Phys. E21 (2012) 1230002, arXiv:1203.5278.

[7] D. de Florian and R. Sassot, Phys.Rev. D69 (2004) 074028, hep-ph/0311227.

[8] M. Hirai, S. Kumano and T.H. Nagai, Phys.Rev. C76 (2007) 065207, arXiv:0709.3038.

[9] K. Eskola, H. Paukkunen and C. Salgado, JHEP 0904 (2009) 065, arXiv:0902.4154.

[10] I. Schienbein et al., Phys.Rev. D80 (2009) 094004, arXiv:0907.2357.

[11] D. de Florian et al., Phys.Rev. D85 (2012) 074028, arXiv:1112.6324.

[12] J.C. Collins and D.E. Soper, Nucl.Phys. B193 (1981) 381.

[13] J.C. Collins and D.E. Soper, Nucl.Phys. B197 (1982) 446.

[14] J.C. Collins, D.E. Soper and G.F. Sterman, Nucl.Phys. B250 (1985) 199.

[15] J.C. Collins, Foundations of perturbative QCD (Cambridge University Press, 2011).

[16] C. Balazs and C.-P. Yuan, Phys.Rev. D56 (1997) 5558, hep-ph/9704258.

[17] F. Landry et al., Phys.Rev. D67 (2003) 073016, hep-ph/0212159.

[18] A.V. Konychev and P.M. Nadolsky, Phys.Lett. B633 (2006) 710, hep-ph/0506225.

[19] A. Guffanti and G. Smye, JHEP 0010 (2000) 025, hep-ph/0007190.

[20] J. Qiu and X. Zhang, Phys.Rev. D63 (2001) 114011, hep-ph/0012348.

[21] S. Tafat, JHEP 0105 (2001) 004, hep-ph/0102237.

[22] A. Kulesza, G.F. Sterman and W. Vogelsang, Phys.Rev. D66 (2002) 014011, hep-ph/0202251.

[23] T. Becher, M. Neubert and D. Wilhelm, JHEP 1202 (2012) 124, arXiv:1109.6027.

[24] M.G. Echevarria et al., (2012), arXiv:1208.1281. 
[25] G.P. Korchemsky and G.F. Sterman, Nucl.Phys. B437 (1995) 415, hep-ph/9411211.

[26] G. Ladinsky and C.-P. Yuan, Phys.Rev. D50 (1994) 4239, hep-ph/9311341.

[27] M. Guzzi and P.M. Nadolsky, (2012), arXiv:1209.1252,

[28] M. Guzzi, P. Nadolsky and B. Wang, (2012, in preparation).

[29] D0 Collaboration, V.M. Abazov et al., Phys.Rev.Lett. 106 (2011) 122001, arXiv:1010.0262.

[30] Z.B. Kang and J. Qiu, Phys.Rev. D77 (2008) 114027, arXiv:0802.2904.

[31] R. Glauber, Phys.Rev. 100 (1955) 242.

[32] V. Gribov, Sov.Phys.JETP 29 (1969) 483.

[33] L. Frankfurt and M. Strikman, Eur.Phys.J. A5 (1999) 293, hep-ph/9812322.

[34] V. Abramovsky, V. Gribov and O. Kancheli, Yad.Fiz. 18 (1973) 595.

[35] L. Frankfurt, V. Guzey and M. Strikman, Phys.Rev. D71 (2005) 054001, hep-ph/0303022.

[36] V. Guzey and M. Strikman, Phys.Lett. B687 (2010) 167, arXiv:0908.1149.

[37] H.-L. Lai et al., Phys.Rev. D82 (2010) 074024, arXiv:1007.2241.

[38] C. Davies and W.J. Stirling, Nucl.Phys. B244 (1984) 337.

[39] C. Davies, B. Webber and W.J. Stirling, Nucl.Phys. B256 (1985) 413.

[40] S. Moch, J. Vermaseren and A. Vogt, Nucl.Phys. B688 (2004) 101, hep-ph/0403192.

[41] P.B. Arnold and M.H. Reno, Nucl.Phys. B319 (1989) 37.

[42] ATLAS Collaboration, G. Aad et al., Phys.Lett. B705 (2011) 415, arXiv:1107.2381.

[43] E.L. Berger, J. Qiu and X. Zhang, Phys.Rev. D65 (2002) 034006, hep-ph/0107309.

[44] Z.B. Kang, J. Qiu and W. Vogelsang, Phys.Rev. D79 (2009) 054007, arXiv:0811.3662.

[45] M. Johnson et al., Phys.Rev. C75 (2007) 035206, hep-ph/0606126.

[46] S. Berge et al., Phys.Rev. D72 (2005) 033015, hep-ph/0410375. 

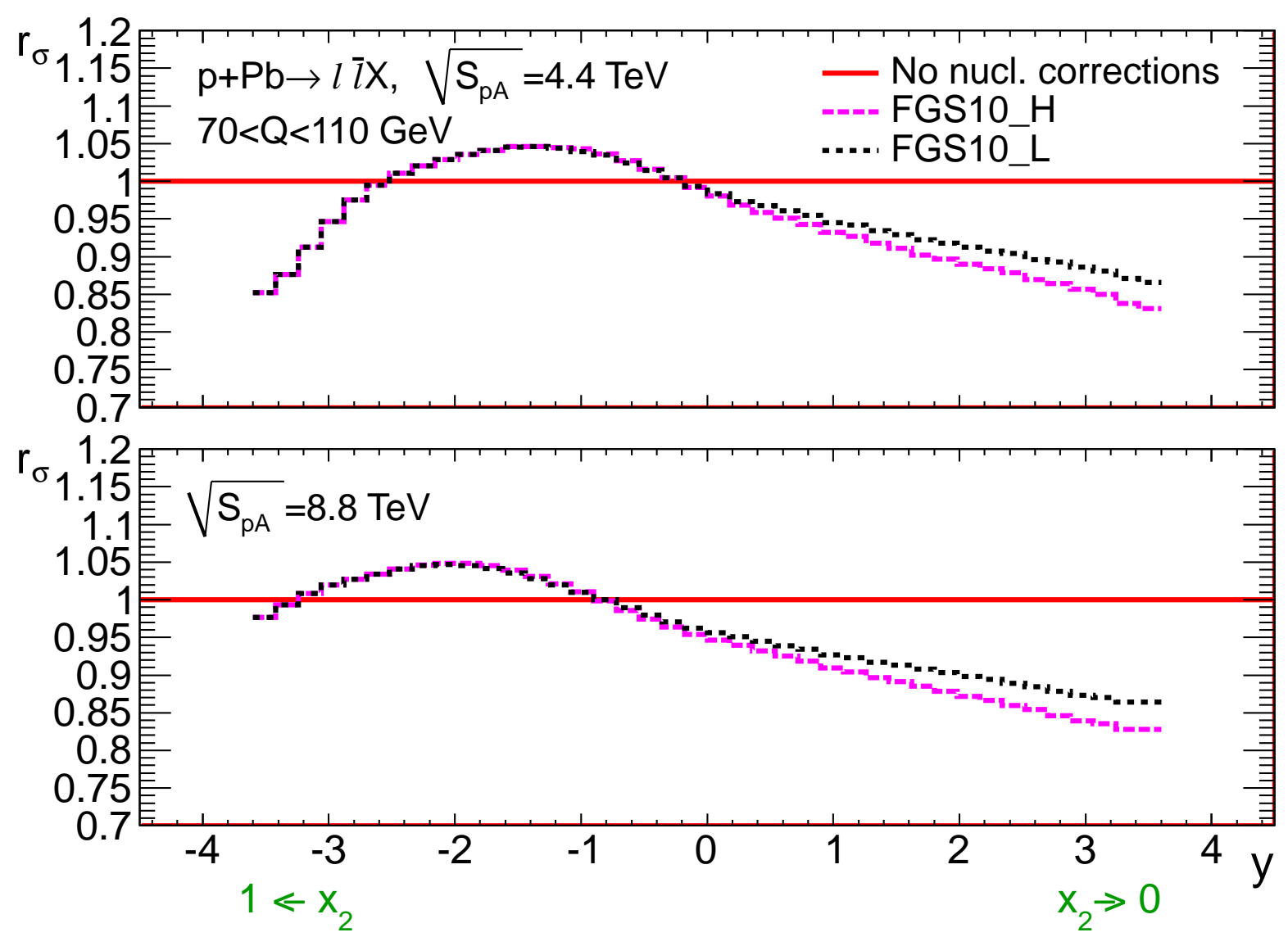

Figure 5: The nuclear correction ratio $r_{\sigma}(y)$, as defined in Eq. (14), plotted for $70<Q<110$ $\mathrm{GeV}$ vs. the lepton pair rapidity $y$ in proton-lead collisions. 

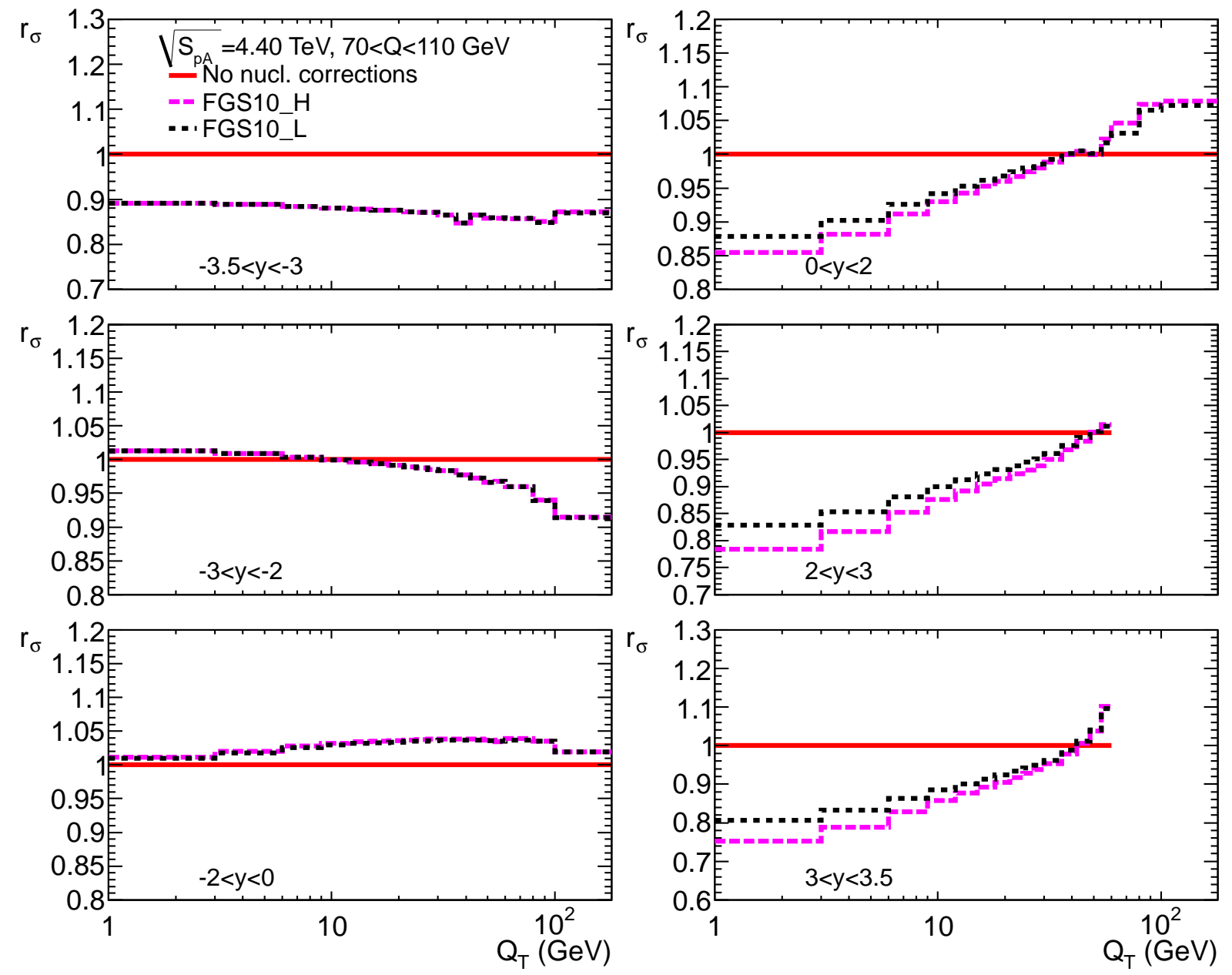

Figure 6: The nuclear correction ratio $r_{\sigma}\left(Q_{T}\right)$ for the $Q_{T}$ distribution and $70<Q<110 \mathrm{GeV}$ in proton-lead collisions at $\sqrt{S_{p A}}=4.4 \mathrm{TeV}$, in six bins of $y$. 

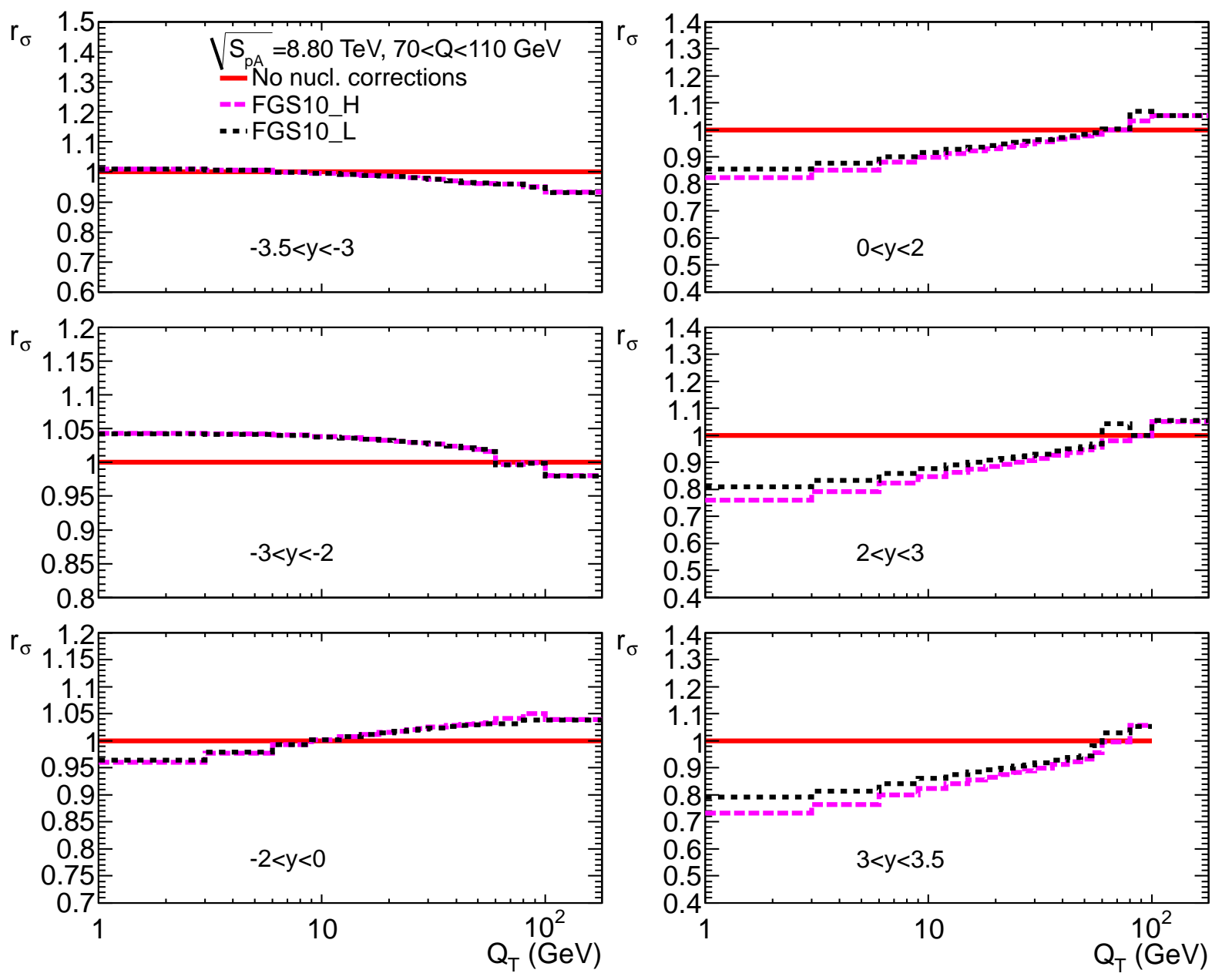

Figure 7: Same as Fig. 6, for $\sqrt{S_{p A}}=8.8 \mathrm{TeV}$. 

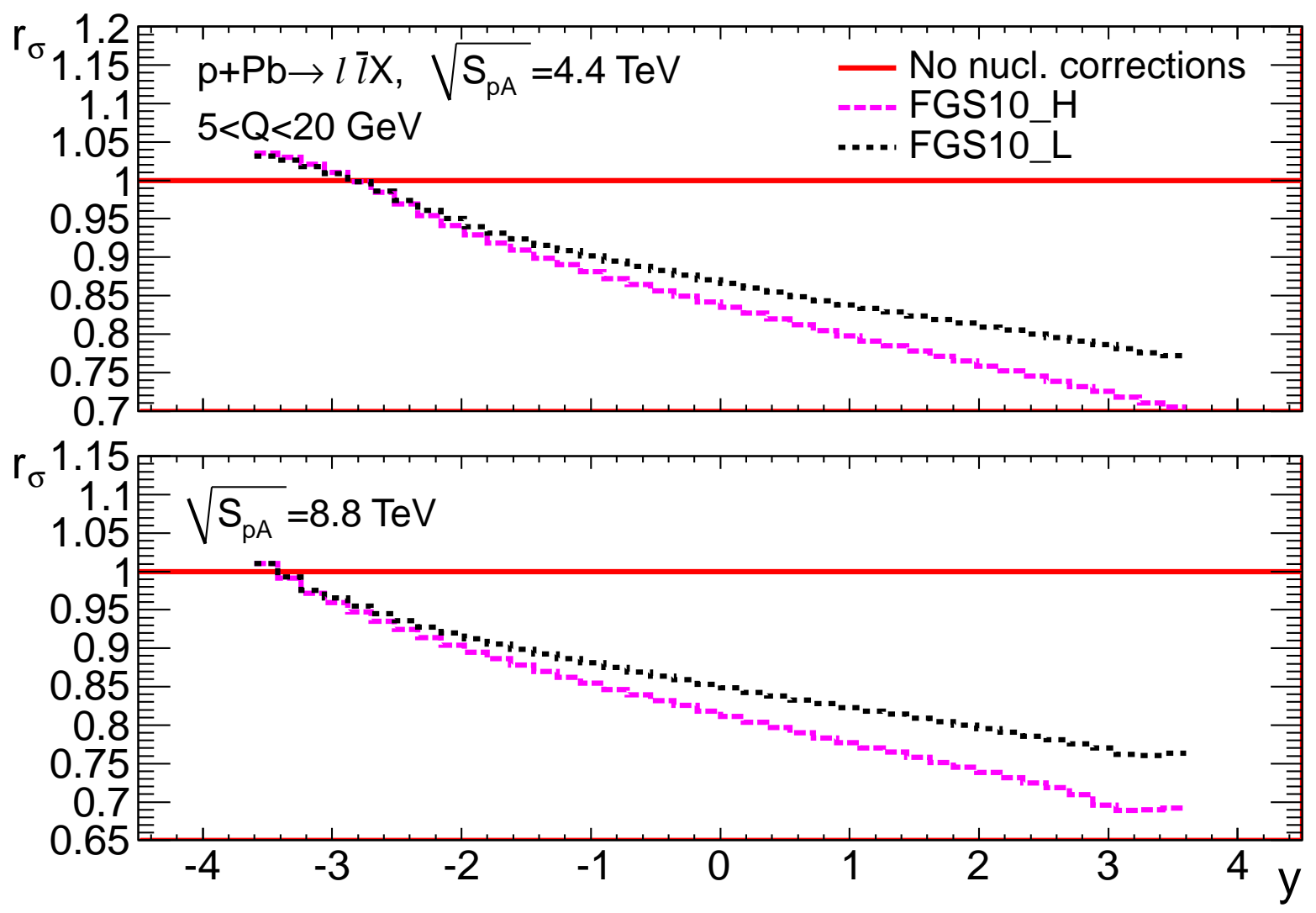

Figure 8: The nuclear correction ratio $r_{\sigma}(y)$, as defined in Eq. (14), plotted for $5<Q<20 \mathrm{GeV}$ vs. the lepton pair rapidity $y$ in proton-lead collisions. 

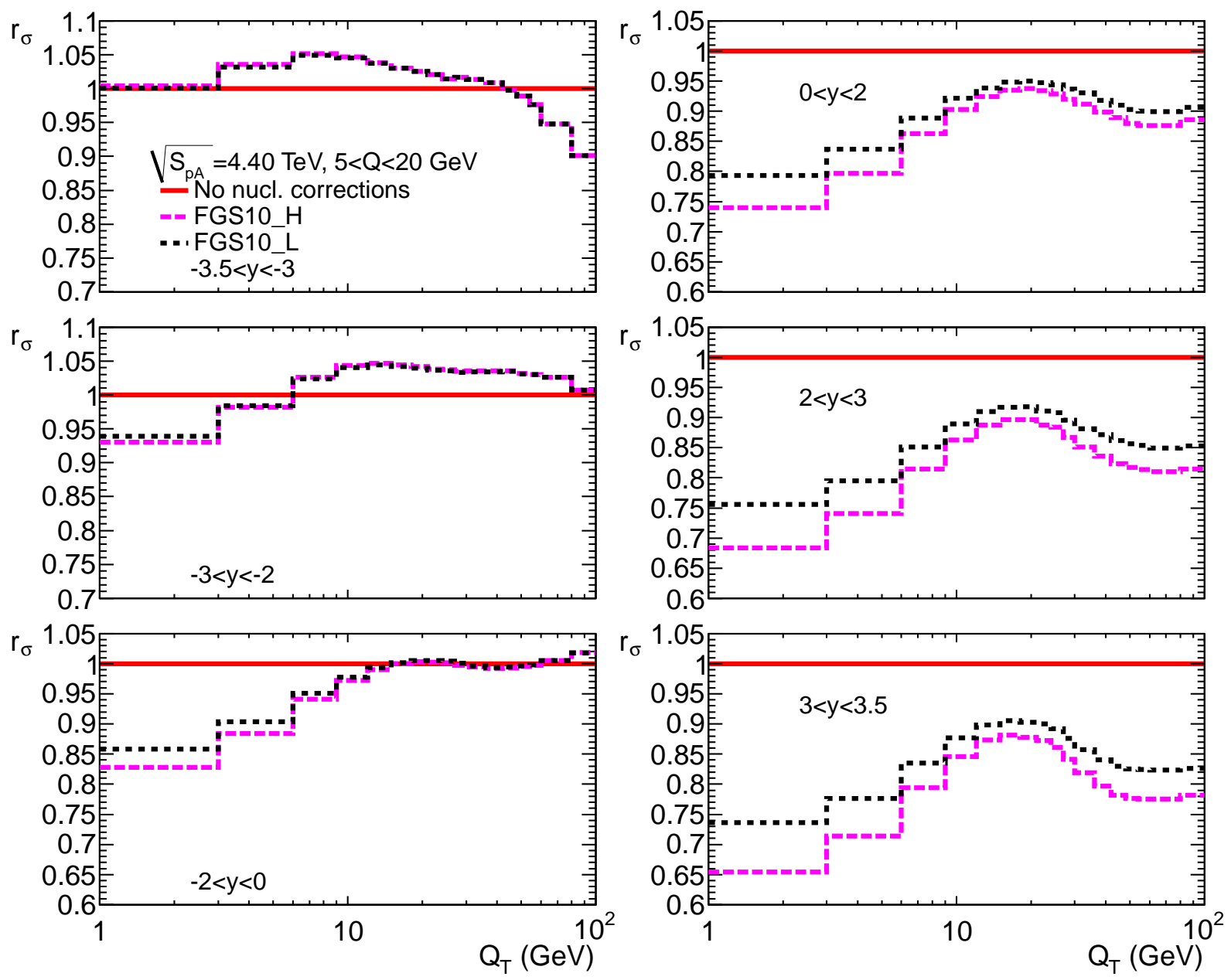

Figure 9: The nuclear correction ratio $r_{\sigma}\left(Q_{T}\right)$ for the $Q_{T}$ distribution and $70<Q<110 \mathrm{GeV}$ in proton-lead collisions at $\sqrt{S_{p A}}=4.4 \mathrm{TeV}$, in six bins of $y$. 

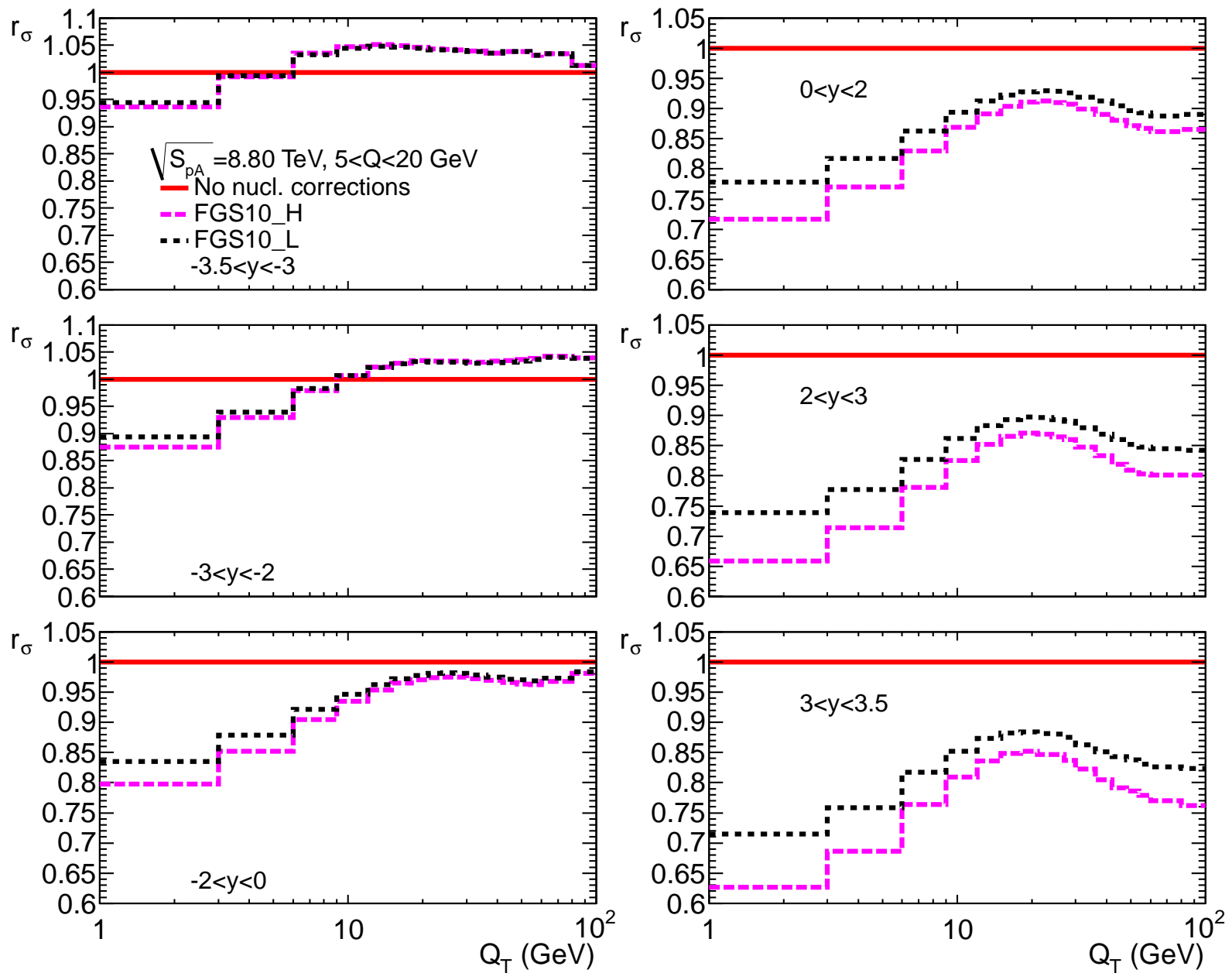

Figure 10: Same as Fig. 9, for $\sqrt{S_{p A}}=8.8 \mathrm{TeV}$. 

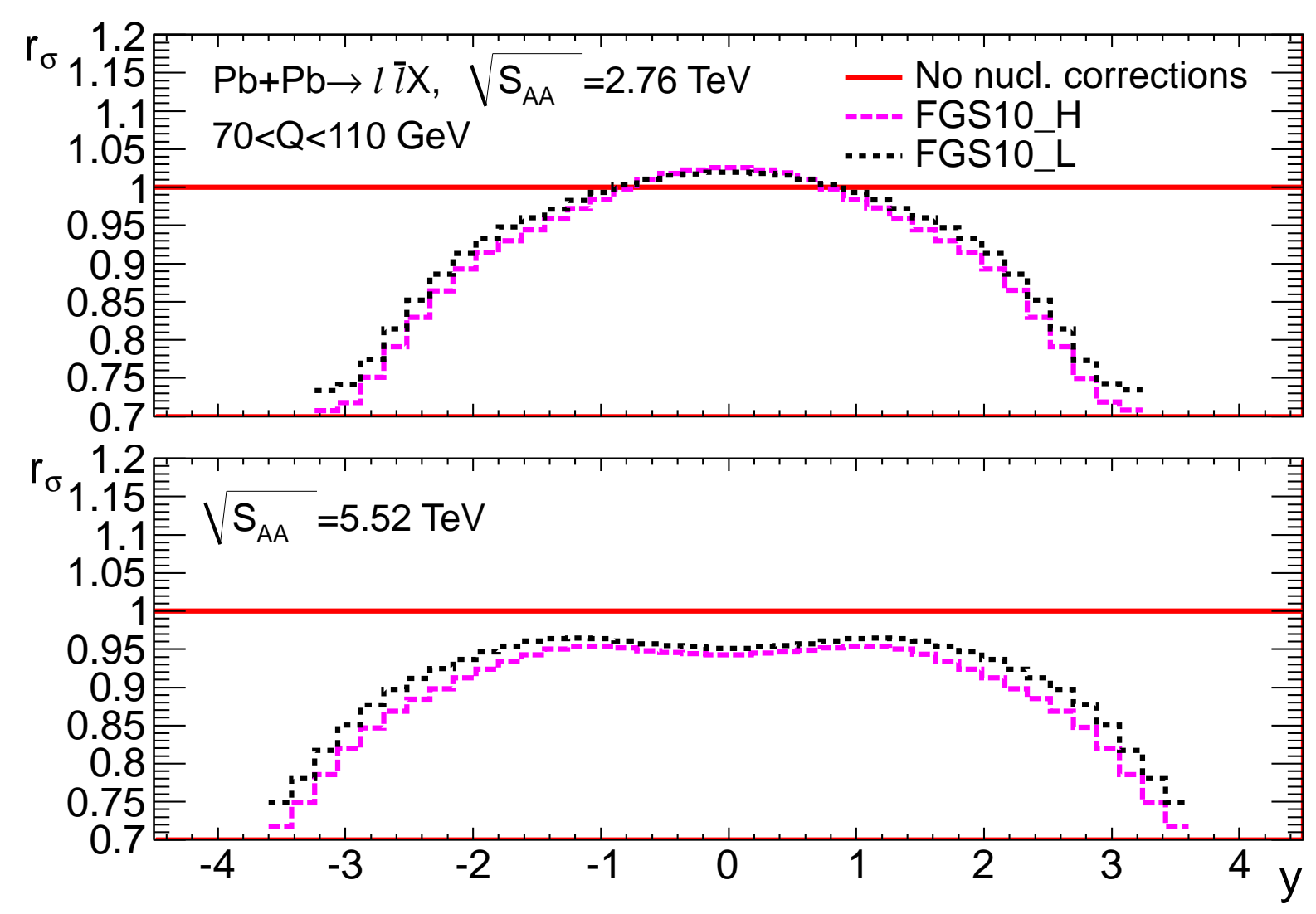

Figure 11: The nuclear correction ratio $r_{\sigma}(y)$, as defined in Eq. (14), plotted for $70<Q<110$ $\mathrm{GeV}$ vs. the lepton pair rapidity $y$ in lead-lead collisions 

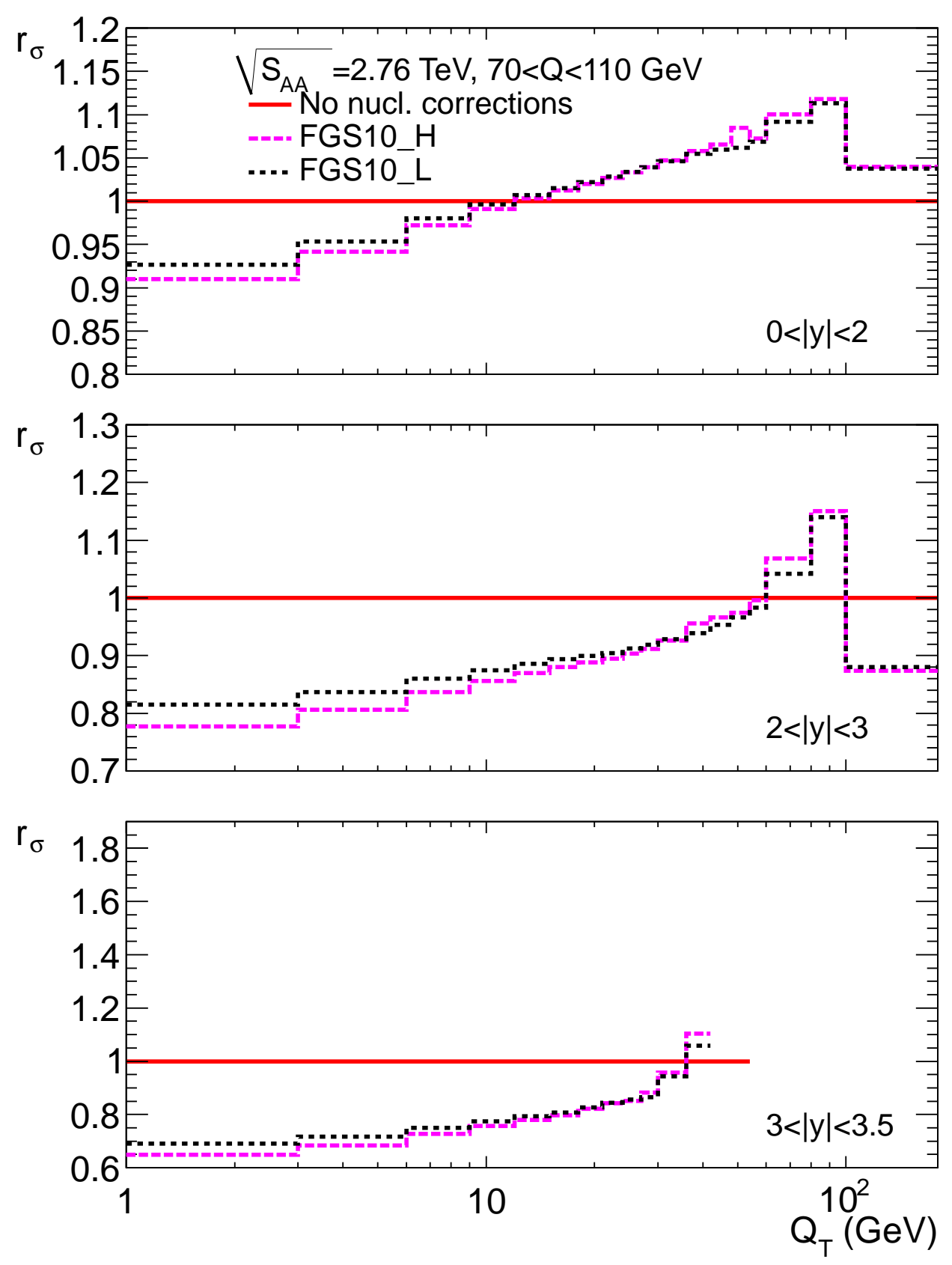

Figure 12: The nuclear correction ratio $r_{\sigma}\left(Q_{T}\right)$ for the $Q_{T}$ distribution and $70<Q<110 \mathrm{GeV}$ in proton-lead collisions at $\sqrt{S_{A A}}=2.76 \mathrm{TeV}$, in three bins of $|y|$. 

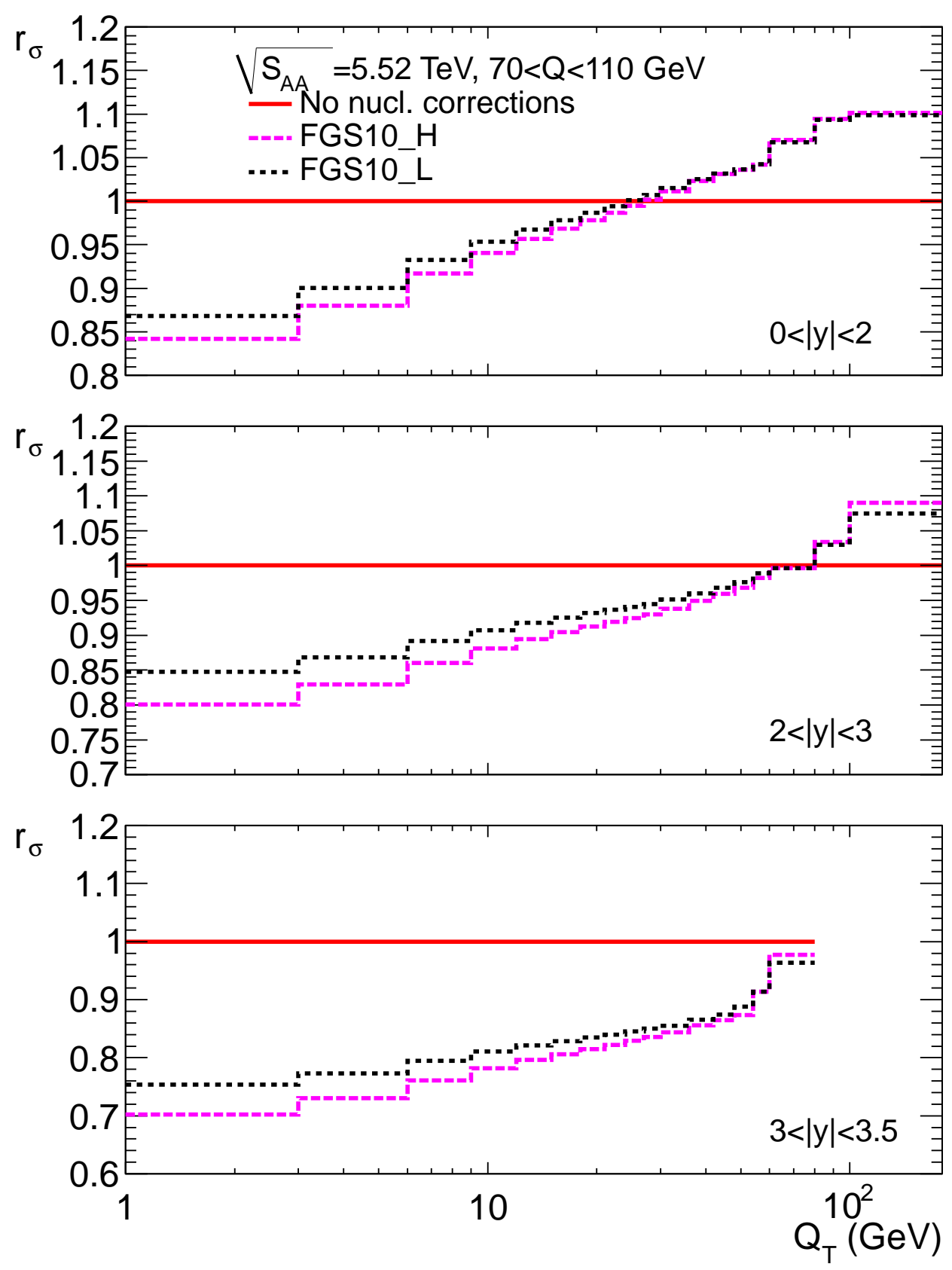

Figure 13: Same as Fig. 12, for $\sqrt{S_{P b P b}}=5.52 \mathrm{TeV}$. 

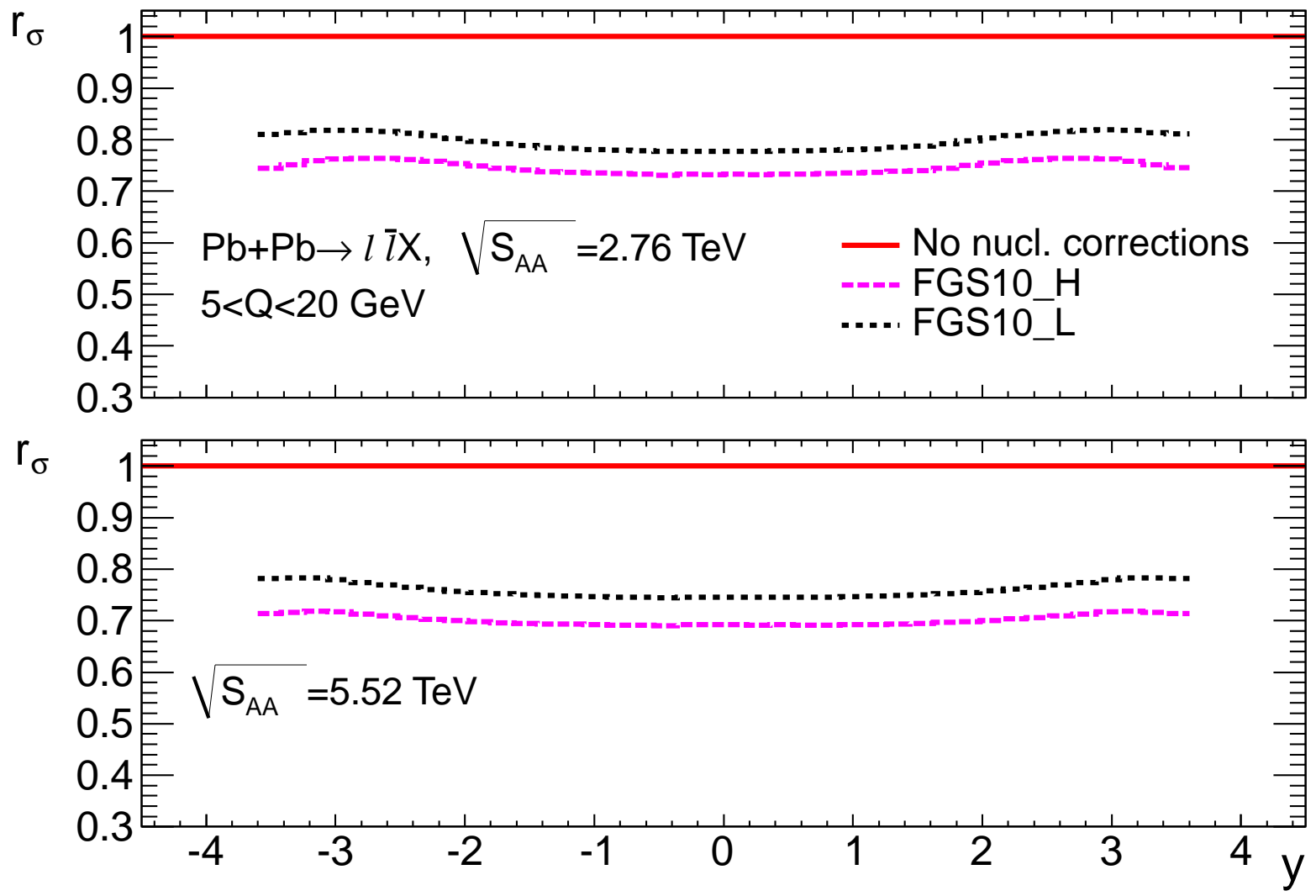

Figure 14: The nuclear correction ratio $r_{\sigma}(y)$, as defined in Eq. (14), plotted for $5<Q<20$ $\mathrm{GeV}$ vs. $y$ in lead-lead collisions 

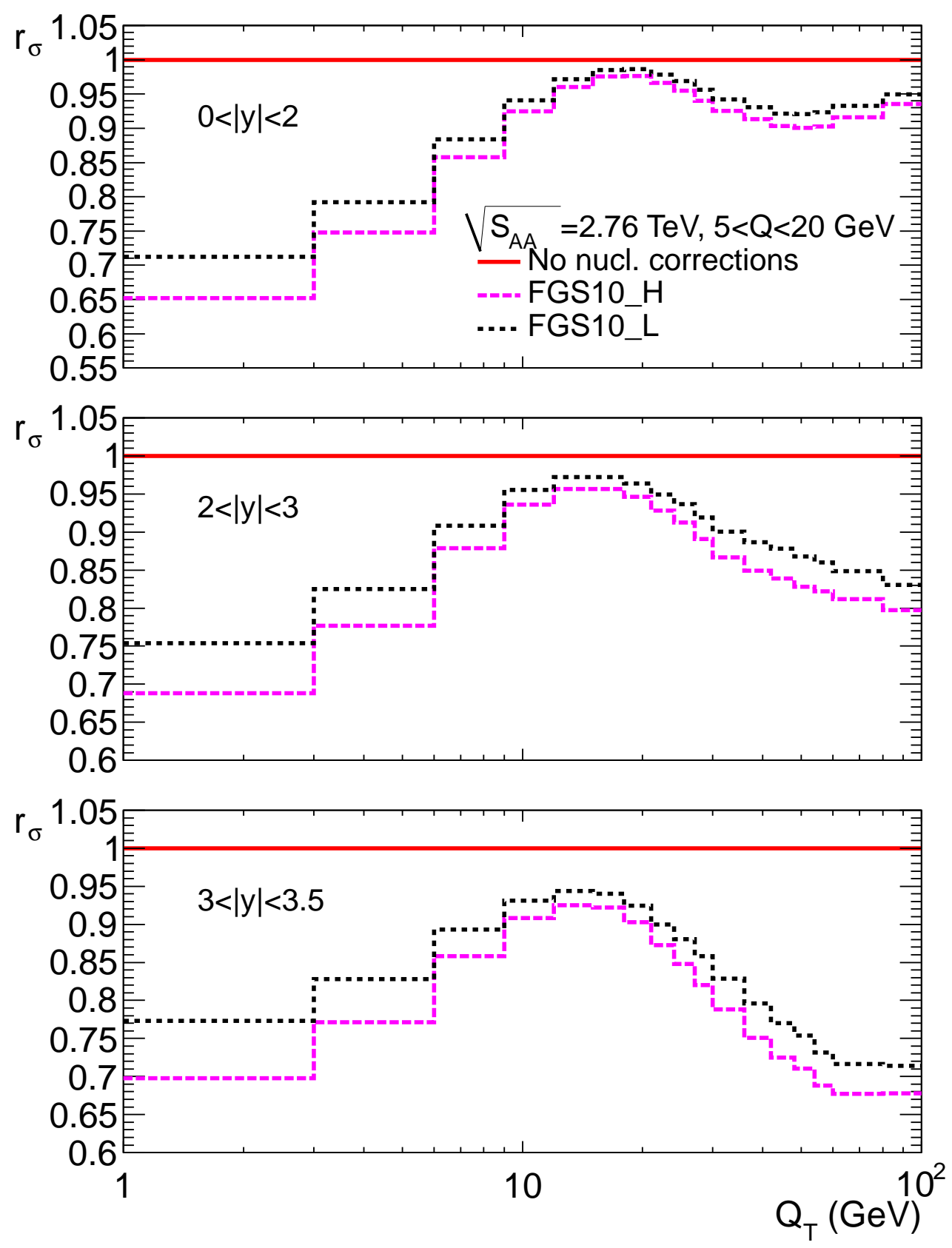

Figure 15: The nuclear correction ratio $r_{\sigma}\left(Q_{T}\right)$ for the $Q_{T}$ distribution and $5<Q<20 \mathrm{GeV}$ in lead-lead collisions at $\sqrt{S_{A A}}=2.76 \mathrm{TeV}$, in three bins of $|y|$. 

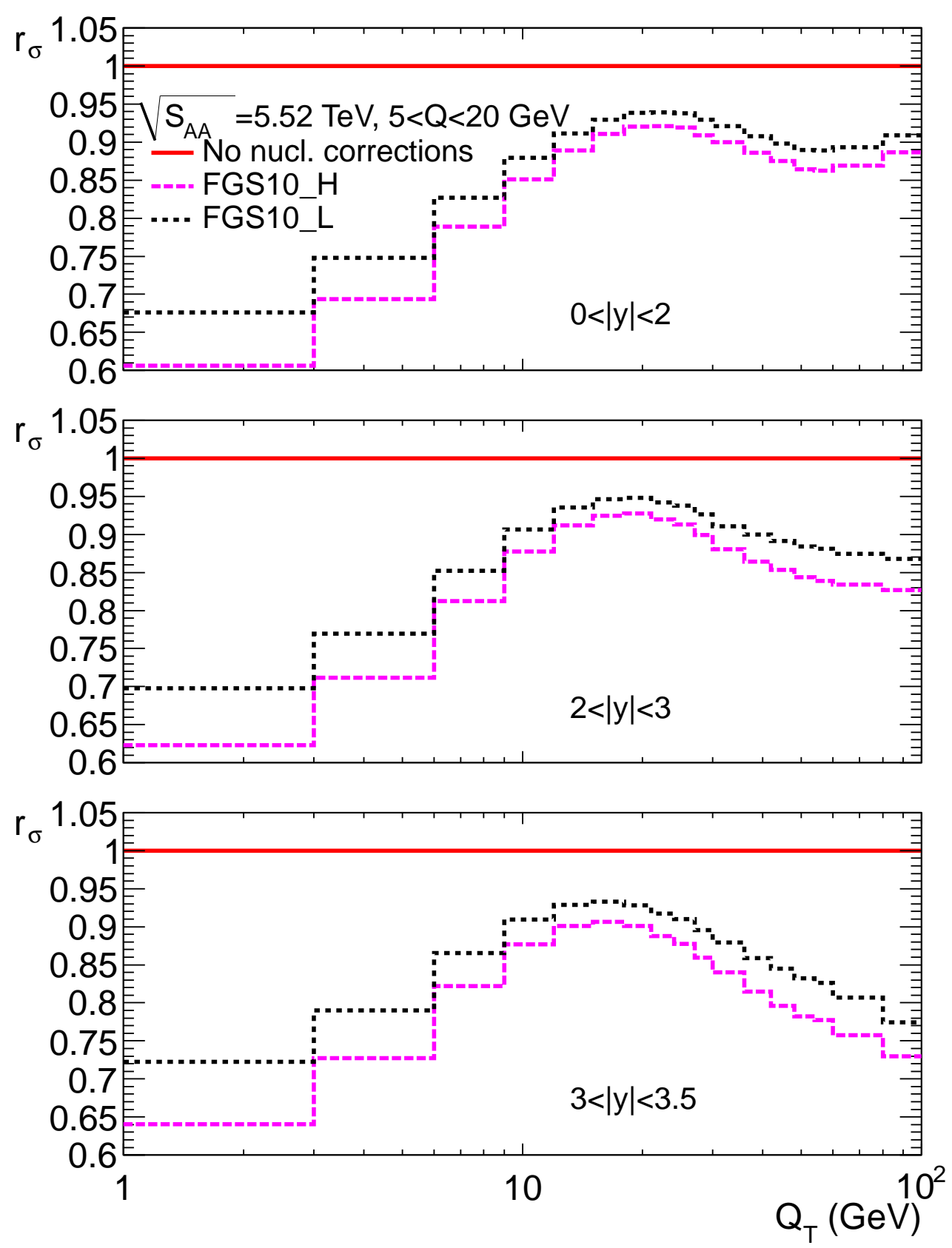

Figure 16: Same as Fig. 15, for $\sqrt{S_{A A}}=5.52 \mathrm{TeV}$. 


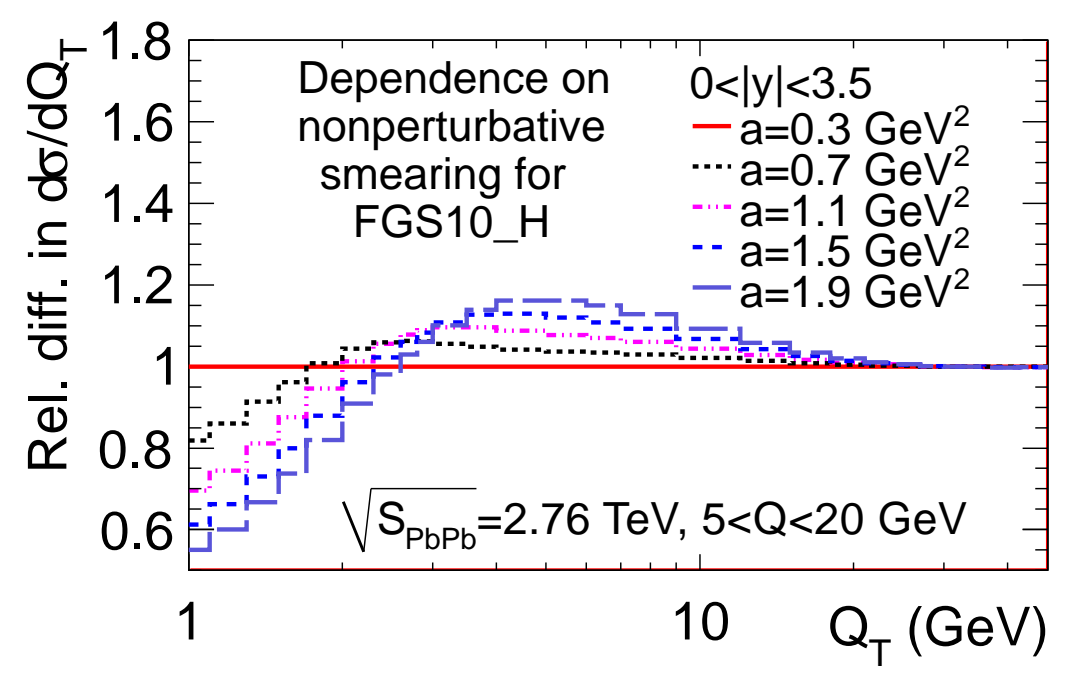

Figure 17: Dependence on nonperturbative smearing of the $Q_{T}$ distribution in lead-lead collisions for $5<Q<20 \mathrm{GeV}$ at $\sqrt{S_{A A}}=2.76 \mathrm{TeV}$.

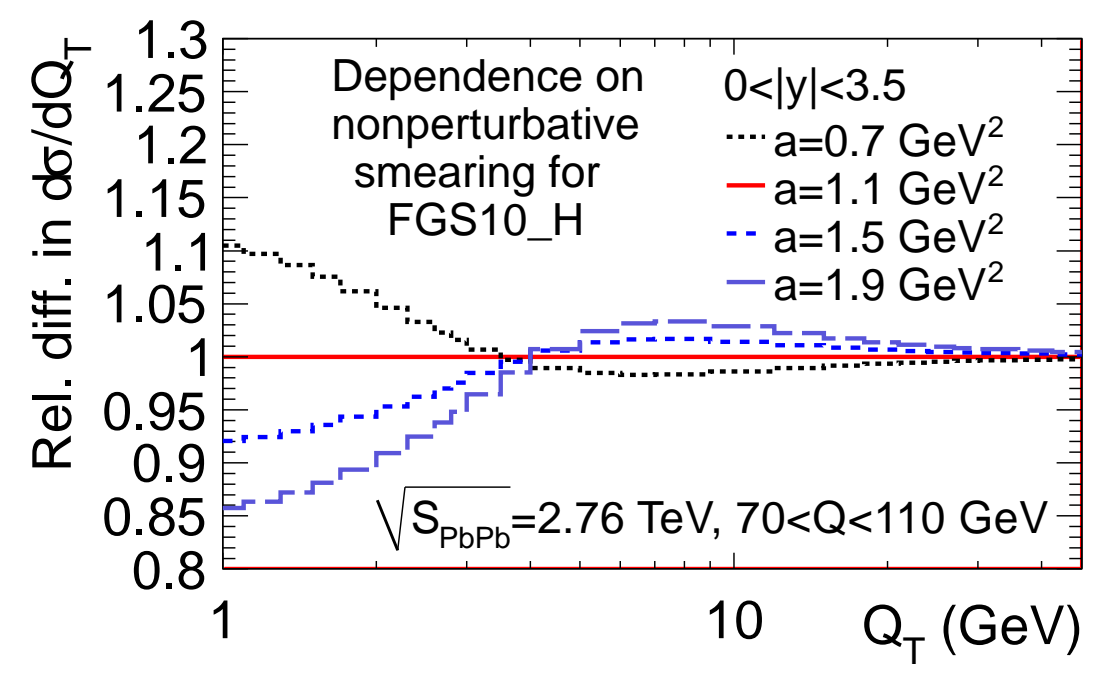

Figure 18: Same as Fig. 17, for $70<Q<110 \mathrm{GeV}$. 